\title{
Sedimentología e Icnología de deltas fluvio-dominados afectados por descargas hiperpícnicas de la Formación Lajas (Jurásico Medio), Cuenca Neuquina, Argentina
}

\author{
Nerina Canale ${ }^{1}$, Juan José Ponce ${ }^{1}$, Noelia B. Carmona ${ }^{1}$, Daniel I. Drittanti ${ }^{2}$, \\ Daniela E. Olivera ${ }^{3}$, Marcelo A. Martínez ${ }^{3}$, Constanza N. Bournod ${ }^{4}$ \\ ${ }^{1}$ CONICET, Universidad Nacional de Río Negro-Instituto de Investigación en Paleobiología y Geología, Avda. Gral. Roca 1242, \\ 8332, General Roca, Río Negro, Argentina. \\ ncanale@unrn.edu.ar;jponce@unrn.edu.ar;ncarmona@unrn.edu.ar \\ 2 Universidad Nacional del Sur, Departamento de Geología, San Juan 670, 8000, Bahía Blanca, Argentina. \\ ddrittanti@gmail.com \\ 3 INGEOSUR-CONICET, Instituto Geológico del Sur, Universidad Nacional del Sur, Departamento de Geología, San Juan 670, 8000, \\ Bahia Blanca, Argentina. \\ danielaolivera2000@yahoo.com.ar;martinez@criba.edu.ar \\ 4 IADO-CONICET, Instituto Argentino de Oceanografía, Camino La Carrindanga Km 7, 8000, Bahía Blanca, Argentina. \\ cbournod@hotmail.com
}

\begin{abstract}
RESUMEN. Existen pocos ejemplos reconocidos de sistemas deltaicos fluvio-dominados afectados por descargas hiperpícnicas. El desarrollo de modelos predictivos en estos sistemas resulta clave para entender la distribución que presentan las facies sedimentarias y para determinar la ubicación de los principales cuerpos arenosos, los cuales constituyen los potenciales reservorios para la acumulación de hidrocarburos. En las áreas de Portada Covunco y Sierra de la Vaca Muerta, la Formación Lajas constituye un excelente ejemplo de una sucesión deltaica fluvio-dominada integrada por depósitos de prodelta y frente deltaico, afectados por descargas hiperpícnicas y reelaborados por acción de oleaje (normal y de tormenta). Los depósitos de prodelta muestran una marcada geometría tabular con alternancia entre fangolitas y areniscas finas con abundante contenido de materia orgánica y desarrollo de una icnofacies de Cruziana empobrecida. Los depósitos de frente deltaico, integrados por fangolitas, areniscas finas a gruesas y conglomerados finos, presentan cuerpos de geometría tabular con desarrollo de las icnofacies de Skolithos y Glossifungites, esta última relacionada con la avulsión de lóbulos deltaicos. La progradación normal del sistema deltaico muestra intervalos estratigráficos integrados por cuerpos lenticulares y tabulares de espesor variable asignados a canales y lóbulos hiperpícnicos. Internamente estos depósitos presentan un pasaje transicional y recurrente entre diferentes estructuras sedimentarias con marcadas variaciones texturales, múltiples superficies de reactivación interna, y abundante contenido de materia orgánica. Los sistemas de canales hiperpícnicos muestran dimensiones y estilos de relleno diferentes. Al pie de las zonas de mayor gradiente, las descargas hiperpícnicas generaron canales mayores con relleno agradacional, mientras que en posiciones de menor gradiente del sistema, se desarrollaron canales de alta sinuosidad. En las zonas donde el flujo hiperpícnico perdió confinamiento se produjo la acumulación de sistemas de lóbulos hiperpícnicos. La presencia de niveles de conglomerados afectados por acción de oleaje coronando los arreglos, marcan los planos de pausa en la sedimentación, en los cuales se desarrollaron ventanas de colonización para el establecimiento de la fauna bentónica.
\end{abstract}

Palabras clave: Formación Lajas, Jurásico, Delta fluvio-dominado, Hiperpicnitas, Icnología. 
ABSTRACT. Sedimentology and Ichnology of fluvio-dominated deltas affected by hyperpycnal discharges Lajas Formation (Middle Jurassic), Neuquén Basin, Argentina. There are few recognized examples of fluvial-dominated deltaic systems affected by hyperpycnal discharges. The development of predictive models in those systems are essential to understand the distribution of sedimentary facies and for determining the location of the main sandy bodies, which constitute the potential hidrocarbon reservoirs. In Portada Covunco and Sierra de la Vaca Muerta areas (Neuquén, Argentina), the Lajas Formation constitutes an excellent example of a deltaic fluvio-dominated succession consisting of prodelta and deltaic front deposits, affected by hyperpycnal discharges and reworked by wave action (storm and normal wave action). Prodelta deposits show a strongly tabular geometry alternating between mudstones and fine sandstones with abundant organic matter and development of an impoverished Cruziana ichnofacies. Deltaic-front deposits are integrated by mudstones, fine to coarse sandstones and fine conglomerates showing tabular geometries and development of the Skolithos and Glossifungites ichnofacies, the later related to deltaic lobe avulsión. Normal progradation of this deltaic system shows stratigraphic intervals consisting of tabular and lenticular bodies of variable thicknesses assigned to hyperpycnal-channel and lobe systems. Internally, these deposits show transitional and recurrent passages between different sedimentary structures with marked textural variations, multiple internal reactivation surfaces and abundant organic matter content. Hyperpycnal channels systems have different dimensions and fill patterns. At the foot of the areas with the greatest gradients, hyperpycnal discharges produced mayor channels with aggradational fill, whereas in areas with lower gradients, high sinuosity channels developed. Hyperpycnal lobes were accumulated in areas where hyperpycnal flows lost confinement. The presence of conglomeratic levels affected by wave action on top of the lobe deposits indicates pauses in sedimentation during which colonization windows could develope, allowing the establishment of the benthic fauna.

Keywords: Lajas Formation, Jurassic, River-dominated delta, Hyperpycnites, Ichnology.

\section{Introducción}

Existen muy pocos ejemplos documentados de sistemas deltaicos fluvio-dominados afectados por descargas hiperpícnicas (Buatois et al., 2011). Consecuentemente el desarrollo de modelos depositacionales integrando datos sedimentológicos e icnológicos en estos sistemas resulta clave, ya que permite tener un detallado control de la distribución que presentan las facies sedimentarias, así como una caracterización de sus principales reservorios. Durante las descargas fluviales, importantes volúmenes de sedimento son transferidos a cuencas marinas y lacustres a través de plumas hipopícnicas, homopícnicas o hiperpícnicas (Mulder y Syvitski, 1995; Syvitsky y Morehead, 1999; Bhattacharya, 2006; Zavala et al., 2006). El desarrollo de uno u otro tipo de proceso de sedimentación en la cuenca depende de una serie de factores; uno de los más significativos es la concentración del flujo debido al volumen de sedimentos transportados durante la descarga fluvial. Mulder y Syvitski (1995) indican que en cuencas marinas, la concentración crítica de sedimentos (Ccs) necesaria para generar una pluma hiperpícnica varía entre $35-45 \mathrm{~kg} \mathrm{~m}^{-3}$, mientras que Parsons et al. (2001), a partir de estudios experimentales, sugieren valores mucho menores $\left(5 \mathrm{~kg} \mathrm{~m}^{-3}\right)$. Cuando la Ccs permanece por debajo de este valor crítico (descargas normales), los sistemas fluviales producen barras y lóbulos are- nosos en su desembocadura, y depósitos de fangolitas en zonas más distales aportadas por decantación y/o floculación desde una pluma hipopícnica. Por el contrario, durante descargas fluviales generadas por eventos de crecida (descargas extraordinarias), la alta concentración de sedimento transportado por el flujo puede superar el valor de Ccs, produciendo corrientes de densidad hiperpícnicas (Mulder y Syvitski, 1995). Las hiperpicnitas se caracterizan por presentar depósitos de espesor variable integrados por un pasaje transicional y recurrente entre diferentes estructuras sedimentarias (Zavala et al., 2006, 2011; Ponce et al., 2008; Ponce, 2009; Ponce y Carmona, 2011). En ambientes marinos, las descargas fluvioderivadas incorporan importantes volúmenes de sedimentos embebidos en agua dulce a la cuenca, produciendo notables variaciones en la salinidad, oxigenación, temperatura y turbidez de la columna de agua (Wetzel, 2008). Estas modificaciones en los parámetros físico-químicos favorecen algunos procesos de sedimentación como son la floculación y la generación de grietas de sinéresis (Schieber et al., 2007; Ponce y Carmona, 2011), pero también ejercen un control muy importante en la diversidad y distribución que presenta la fauna bentónica (MacEachern et al., 2005; Buatois et al., 2011; Carmona y Ponce, 2011).

En las áreas de Portada Covunco y Sierra de la Vaca Muerta, la Formación Lajas (Jurásico Medio) 
conforma una sucesión grano estrato creciente que supera los $600 \mathrm{~m}$ de espesor, donde depósitos de fangolitas de prodelta son reemplazadas, en transición, por areniscas y conglomerados de frente deltaico. Zavala y González (2001) interpretan estos depósitos como sistemas deltaicos afectados por descargas hiperpícnicas y parcialmente reelaborados por corrientes de marea y oleaje, mientras que McIlroy et al. (2005) le asignan un origen deltaico con dominio de la acción de oleaje sobre la de las mareas. Estudios recientemente realizados muestran un dominio de estructuras tractivas unidireccionales (óndulas de corriente y estratificación entrecruzada tangencial) con abundante contenido de materia orgánica principalmente acumulada en sus caras de avalancha, que permiten reconocer una señal fluvial muy marcada para estos depósitos deltaicos (Ponce et al., 2012). Este caso de estudio combina observaciones sedimentológicas e icnológicas en la caracterización de un sistema deltaico, ofreciendo una interpretación alternativa a depósitos que han sido tradicionalmente considerados como deltas dominados por marea. Por otra parte, los depósitos de la Formación Lajas han sido utilizados como análogos de los depósitos jurásicos del Mar del Norte (McIlroy, 2004), los cuales constituyen importantes reservorios, por lo que esta reinterpretación tiene considerables implicancias para el modelado de estos sistemas. Teniendo en cuenta lo anteriormente expuesto, los objetivos del trabajo son: 1. documentar y describir las principales asociaciones de facies sedimentarias y geometrías que integran los depósitos deltaicos de la Formación Lajas en las localidades de Portada Covunco y Sierra de la Vaca Muerta, discutiendo los procesos y mecanismos de sedimentación que dan lugar a una acumulación alternante entre depósitos de prodelta, frente deltaico y corrientes de densidad hiperpícnicas; 2. describir el contenido icnológico para cada uno de los subambientes reconocidos, discutiendo los probables parámetros paleoecológicos que controlaron la distribución de las trazas fósiles; $y$ 3. presentar un modelo depositacional que explique la distribución espacial y temporal que muestran los diferentes elementos arquitecturales que integran esta sucesión.

\section{Marco Geológico}

La Cuenca Neuquina se localiza al centro-oeste de la Argentina (Fig. 1A) abarcando un área de más de $200.000 \mathrm{~km}^{2}$ (Yrigoyen, 1991). Esta cuenca ha sido definida como una cuenca de retroarco multiepisódica, desarrollada sobre corteza continental en el margen oeste de Sudamérica y originada por el colapso termo-tectónico detrás de un arco magmático estacionario durante el Triásico Tardío (Mpodozis y Ramos, 1989). Sus depósitos fueron acumulados en tres etapas principales (Fig. 1B), dando lugar a un registro estratigráfico continuo de aproximadamente $7.000 \mathrm{~m}$ de espesor, integrado por rocas sedimentarias acumuladas en medios marinos y continentales del Triásico superior al Paleoceno (Arregui et al., 2011). Durante la primera etapa (Triásico Tardío-Jurásico Temprano), se produce la acumulación de materiales volcánicos y volcanoclásticos del Grupo Precuyo, cuyos espesores y distribución regional están controlados por la presencia de hemigrábenes (Gulisano, 1981; Gulisano et al., 1984). La segunda etapa (Jurásico Temprano-Jurásico Tardío), se encuentra integrada por depósitos marinos y continentales, mayormente de origen clástico, correspondientes a los grupos Cuyo y Lotena (Gulisano, 1981). Zavala y González (2001) sugieren que estos depósitos se acumularon sobre un relieve irregular debido a cambios tectónicos y del nivel de base, los cuales habrían provocado un marcado control en la distribución de facies y geometría de las unidades. La tercera etapa (Jurásico Tardío-Cretácico Tardío) se compone por una espesa sucesión marina y continental correspondiente a los grupos Mendoza, Rayoso y Neuquén. Estos depósitos son los que presentan la mayor distribución areal en la cuenca y su arreglo interno resulta por cambios del nivel de base y, en menor medida, por la actividad tectónica (Gulisano et al., 1984; Legarreta y Gulisano, 1989; Gulisano y Gutiérrez Pleimling, 1995). Hacia el Cretácico Tardío, el cambio en el régimen de subsidencia en el margen continental activo, produjo el ascenso del arco magmático y su progresiva transformación en una cuenca de antepaís, que perduró hasta el Cenozoico (Spaletti et al., 2005; Tunik et al., 2010).

Dentro de este contexto evolutivo, el Grupo Cuyo (Jurásico Temprano a Medio) representa el primer episodio de sedimentación marina generalizada en la Cuenca Neuquina (Groeber, 1946). En las áreas de Portada Covunco y Sierra de la Vaca Muerta, los depósitos del Grupo Cuyo constituyen una sucesión sedimentaria que supera los $600 \mathrm{~m}$ de espesor (Zavala y González, 2001; Zavala, 2002). La 


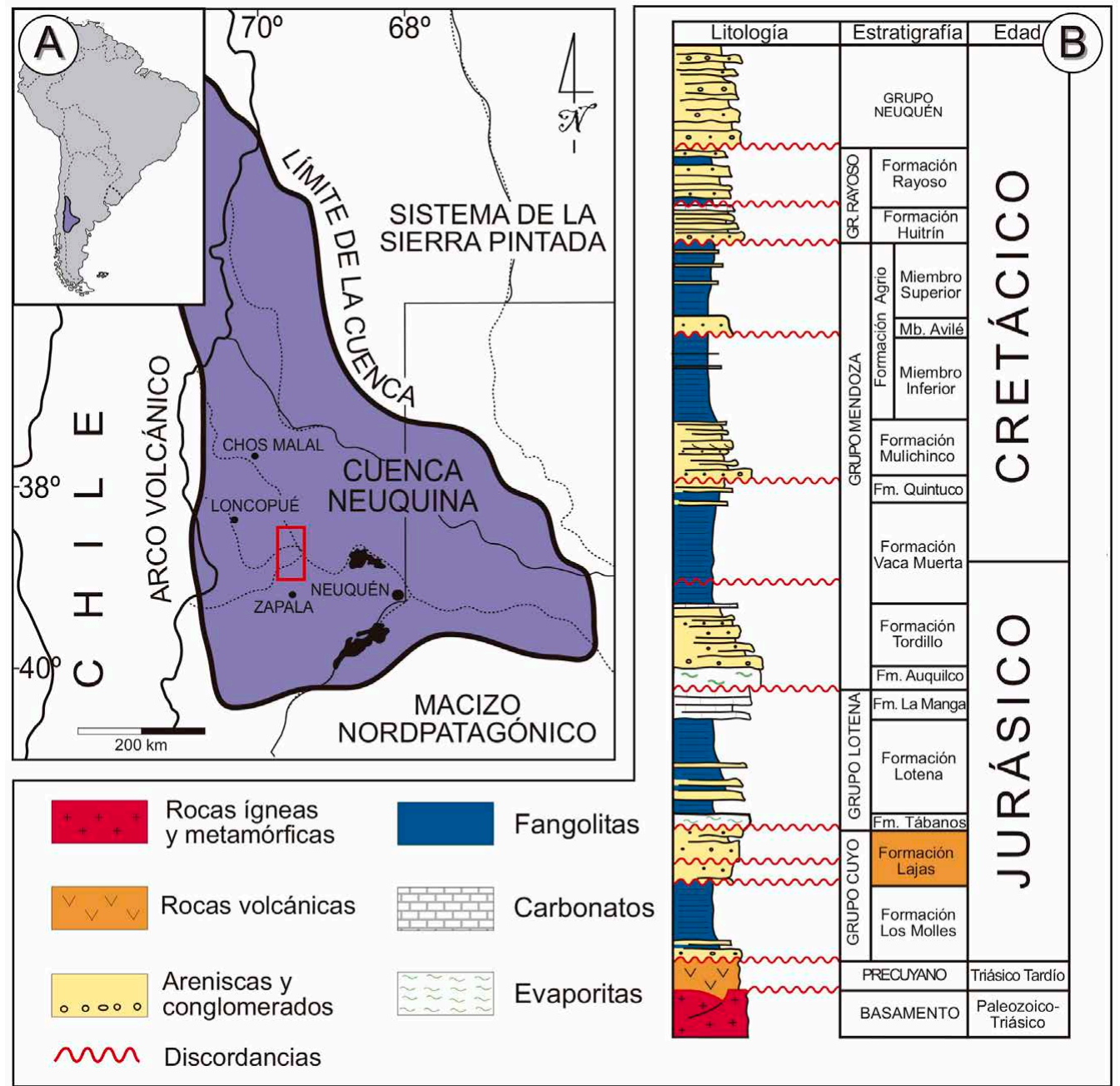

FIG. 1. A. Mapa de la Cuenca Neuquina indicando el área de estudio (recuadro rojo); B. Columna estratigráfica de la Cuenca Neuquina indicando la ubicación de la Formación Lajas (rectángulo naranja) (modificado de Zavala y González, 2001).

sección basal del grupo está representada por lutitas negras que gradan hacia areniscas finas correspondientes a la Formación Los Molles, acumuladas en sistemas deltaicos con incursiones turbidíticas asociadas (Leanza y Hugo, 2001). Estos depósitos son sucedidos en transición por cuerpos tabulares y lenticulares correspondientes a la Formación Lajas, los cuales están integrados por areniscas, fangolitas y en menor medida conglomerados, que fueron asignados a sistemas deltaicos afectados por descargas hiperpícnicas y parcialmente reelaborados por corrientes de marea y oleaje (Zavala y González, 2001), o a deltas dominados por oleaje más que por marea (McIlroy et al., 2005).

\section{Sedimentología, distribución de trazas fósiles y ambiente depositacional}

La Formación Lajas en las localidades analizadas de Portada Covunco y Sierra de la Vaca Muerta conforma una sucesión grano-estrato creciente integrada por depósitos de fangolitas, areniscas gruesas a finas, y de manera subordinada, conglomerados acumulados en cuerpos de geometría tabular y lenticular (Tabla 1, Fig. 2). Tres asociaciones de facies principales han sido reconocidas en estos depósitos: 1. asociaciones de facies de prodelta distal y proximal; 2 . asociaciones de facies de frente deltaico distal y proximal; y 3. asociaciones de facies de canal y lóbulo hiperpícnico. 


\begin{tabular}{|c|c|c|c|c|c|c|c|c|c|c|c|}
\hline \multirow{3}{*}{ Facies } & \multirow{3}{*}{ Litología } & \multirow{3}{*}{$\begin{array}{l}\text { Estructura } \\
\text { sedimentaria }\end{array}$} & \multirow{3}{*}{$\begin{array}{l}\text { Trazas } \\
\text { fósiles }\end{array}$} & \multirow{3}{*}{ Geometría } & \multirow{3}{*}{ Origen } & \multicolumn{6}{|c|}{ Ocurrencia } \\
\hline & & & & & & \multicolumn{2}{|c|}{ Prodelta } & \multicolumn{2}{|c|}{ Frente deltaico } & \multicolumn{2}{|c|}{ Hiperpicnita } \\
\hline & & & & & & distal & proximal & distal & proximal & canal & $\begin{array}{c}\begin{array}{c}\text { lóbulo/ } \\
\text { albardón }\end{array} \\
\end{array}$ \\
\hline Mm & Fangolita & Maciza & $\begin{array}{l}\text { Planolites, Chondrites, } \\
\text { Teichichnus }\end{array}$ & Tabular & $\begin{array}{l}\text { Floculación desde una pluma hipopícnica } \\
\text { y/o hiperpícnica con abundante contenido } \\
\text { de materia orgánica. }\end{array}$ & & & & & & \\
\hline MI & $\begin{array}{l}\text { Fangolita con } \\
\text { materia orgánica }\end{array}$ & $\begin{array}{l}\text { Laminación } \\
\text { paralela }\end{array}$ & $\begin{array}{l}\text { Planolites, Chondrites, } \\
\text { Teichichnus }\end{array}$ & Tabular & $\begin{array}{l}\text { Decantación desde una pluma hipopícnica } \\
\text { y/o hiperpícnica con abundante contenido } \\
\text { de materia orgánica. }\end{array}$ & & & & & & \\
\hline Sm & $\begin{array}{l}\text { Arenisca fina a } \\
\text { mediana con materia } \\
\text { orgánica }\end{array}$ & Maciza & Ophiomorpha & $\begin{array}{l}\text { Tabular y } \\
\text { lenticular }\end{array}$ & $\begin{array}{l}\text { Depósitos producidos por: 1) agradación } \\
\text { continua desde una corriente de densidad } \\
\text { (proceso físico) y/o 2) por bioturbación } \\
\text { (proceso biológico). }\end{array}$ & & & & & & \\
\hline Spcb & $\begin{array}{l}\text { Arenisca fina a } \\
\text { gruesa con materia } \\
\text { orgánica }\end{array}$ & $\begin{array}{l}\text { Estratificación } \\
\text { entrecruzada tabular } \\
\text { planar }\end{array}$ & $\begin{array}{c}\text { Arenicolites, } \\
\text { Diplocraterion, } \\
\text { Ophiomorpha, } \\
\text { Rhizocorallium Skolithos }\end{array}$ & $\begin{array}{l}\text { Tabular y } \\
\text { lenticular }\end{array}$ & $\begin{array}{l}\text { Migración de formas de lecho } 2 \mathrm{D} \text { de gran } \\
\text { escala producidas por corrientes } \\
\text { unidireccionales diluidas y turbulentas. }\end{array}$ & & & & & & \\
\hline Stcb & $\begin{array}{l}\text { Arenisca fina a } \\
\text { mediana }\end{array}$ & $\begin{array}{c}\text { Estratificación } \\
\text { entrecruzada } \\
\text { tangencial y en artesa }\end{array}$ & $\begin{array}{l}\text { Macaronichnus, } \\
\text { Dactyloidites, Curvolithus, } \\
\text { Trazas de equilibrio/ } \\
\text { escape }\end{array}$ & $\begin{array}{l}\text { Tabular y } \\
\text { lenticular }\end{array}$ & $\begin{array}{l}\text { Migración de formas de lecho } 3 \mathrm{D} \text { de gran } \\
\text { escala producidas por corrientes } \\
\text { unidireccionales diluidas y turbulentas. }\end{array}$ & & & & & & \\
\hline SI & $\begin{array}{l}\text { Arenisca fina a } \\
\text { mediana }\end{array}$ & $\begin{array}{l}\text { Laminación paralela } \\
\text { con lineación parting }\end{array}$ & $\begin{array}{l}\text { Ophiomorpha, } \\
\text { Skolithos }\end{array}$ & Tabular & $\begin{array}{l}\text { Procesos tractivos o de } \\
\text { decantación en tracción- } \\
\text { régimen de flujo. }\end{array}$ & & & & & & \\
\hline Scd & $\begin{array}{l}\text { Arenisca fina a } \\
\text { mediana }\end{array}$ & Dunas escalantes & - & Lenticular & $\begin{array}{l}\text { Procesos de tracción-decantación } \\
\text { generados por corrientes de densidad } \\
\text { unidireccionales. }\end{array}$ & & & & & & \\
\hline $\mathrm{Sr}$ & $\begin{array}{c}\text { Arenisca fina con } \\
\text { materia orgánica }\end{array}$ & $\begin{array}{l}\text { Laminación } \\
\text { ondulítica y } \\
\text { lenticular }\end{array}$ & $\begin{array}{c}\text { Teichichnus, } \\
\text { Planolites, Gyrochorte }\end{array}$ & $\begin{array}{l}\text { Tabular y } \\
\text { lenticular }\end{array}$ & $\begin{array}{l}\text { Tracción producida por corrientes } \\
\text { unidireccionales diluidas sobre la } \\
\text { superficie no cohesiva de los sedimentos } \\
\text { del fondo. }\end{array}$ & & & & & & \\
\hline Srw & $\begin{array}{l}\text { Arenisca fina con } \\
\text { materia orgánica }\end{array}$ & $\begin{array}{c}\text { Laminación } \\
\text { ondulítica simétrica }\end{array}$ & $\begin{array}{l}\text { Gyrochorte, } \\
\text { Skolithos }\end{array}$ & $\begin{array}{l}\text { Tabular y } \\
\text { lenticular }\end{array}$ & $\begin{array}{l}\text { Interacción de una corriente oscilatoria } \\
\text { con el lecho arenoso. }\end{array}$ & & & & & & \\
\hline $\mathrm{Cg}$ & $\begin{array}{l}\text { Conglomerado fino } \\
\text { a medio }\end{array}$ & $\begin{array}{l}\text { Macizo o con } \\
\text { laminación difusa }\end{array}$ & $\begin{array}{l}\text { Ophiomorpha, } \\
\quad \text { Skolithos }\end{array}$ & $\begin{array}{l}\text { Tabular y } \\
\text { lenticular }\end{array}$ & $\begin{array}{l}\text { Transporte como carga de lecho producido } \\
\text { por corrientes de densidad. }\end{array}$ & & & & & & \\
\hline Cgrw & $\begin{array}{l}\text { Conglomerado fino } \\
\text { con materia orgánica }\end{array}$ & $\begin{array}{l}\text { Óndulas y } \\
\text { megaóndulas }\end{array}$ & $\begin{array}{c}\text { Ophiomorpha, Skolithos, } \\
\text { Rhizocorallium, } \\
\text { Trazas de equilibrio/ } \\
\text { escape } \\
\end{array}$ & $\begin{array}{l}\text { Tabular y } \\
\text { lenticular }\end{array}$ & $\begin{array}{l}\text { Corrientes oscilatorias generadas durante } \\
\text { eventos de tormenta que lavan la } \\
\text { superficie de los depósitos concentrando el } \\
\text { material más grueso. }\end{array}$ & & & & & & \\
\hline Gs & Arenisca fina & Grietas de sinéresis & - & Lenticular & Cambios de salinidad. & & & & & & \\
\hline
\end{tabular}




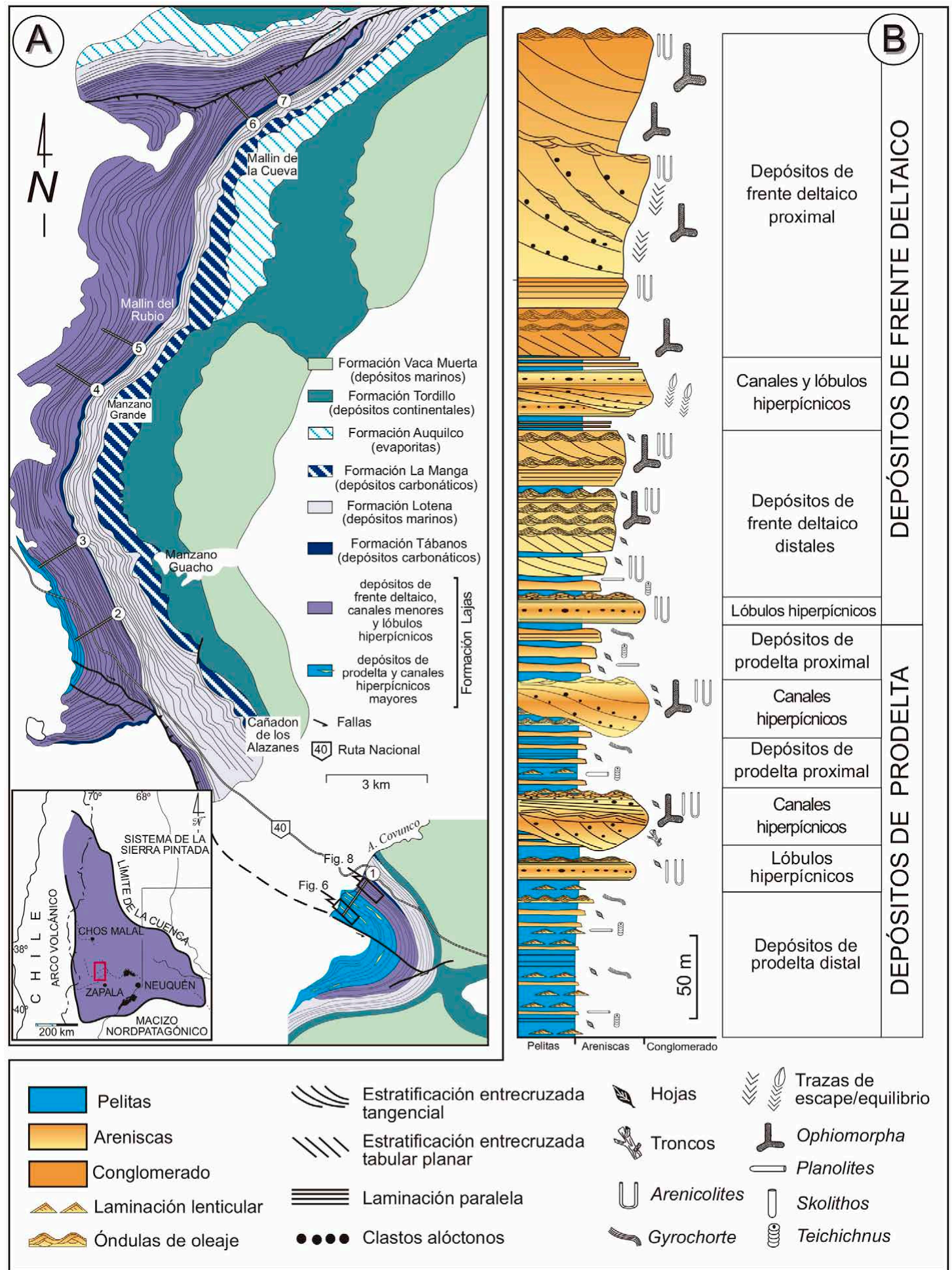

FIG. 2. A. Mapa geológico del área de estudio indicando los ambientes y subambientes que integran la Formación Lajas (modificado de Zavala y González, 2001); B. Sección esquemática mostrando el arreglo progradante del sistema deltaico y la ubicación de los sistemas de canales y lóbulos hiperpícnicos. Los rectángulos negros indican la ubicación de las figuras 6 y 8. 


\subsection{Asociaciones de facies de prodelta distal y proximal}

Las asociaciones de facies de prodelta distal y proximal están integradas por fangolitas y areniscas finas, dispuestas en cuerpos de geometría tabular con arreglo general grano-estrato creciente (Fig. 3A). Estas asociaciones de facies se encuentran muy bien expuestas en la localidad de Portada Covunco, donde alcanzan un espesor de 207 m. (Fig. 2).

\subsubsection{Asociación de facies de prodelta distal}

Consiste de una alternancia centimétrica de fangolitas macizas y/o laminadas con grietas de sinéresis que, de manera subordinada, incluye areniscas finas con laminación ondulosa y lenticular, con abundante contenido de materia orgánica particulada (Fig. 3B). A menudo, las caras de avalancha de las óndulas de corriente se encuentran tapizadas por delgados niveles de materia orgánica y fango. Los vectores de paleocorriente indican una dispersión de sedimentos hacia el NE-NW. Esta facies muestra intervalos no bioturbados o moderadamente bioturbados con Teichichnus, Planolites y Chondrites en las fracciones más fangosas (Fig. 3B, C). La presencia de una suite monoespecífica de Chondrites, indicaría condiciones disaeróbicas producidas por la rápida acumulación de materia orgánica y fango (Savrda y Bottjer, 1987, 1989). Interpretación: el dominio de fangolitas sobre las areniscas finas con laminación ondulosa y lenticular, sugiere que estos depósitos se acumularon en un ambiente de baja energía dominado por procesos de decantación (con floculación de arcilla), desde una pluma hipopícnica con abundante contenido de materia orgánica. La presencia de grietas de sinéresis y el contenido icnológico en esta facies, integrado por una suite de organismos depositívoros característica de una icnofacies de Cruziana empobrecida, sugieren condiciones de estrés, posiblemente relacionadas con cambios en la salinidad y turbidez del medio.

\subsubsection{Asociación de facies de prodelta proximal}

Se encuentra integrada por una alternancia entre areniscas medianas a finas, y fangolitas macizas o laminadas dispuestas en cuerpos de geometría tabular de hasta $20 \mathrm{~cm}$ de espesor (Fig. 3D). Internamente los bancos de areniscas pueden ser macizos o presentar estructuras con laminación paralela, óndulas de corriente y óndulas simétricas (Fig. 3E) con abundante contenido de materia orgánica (particulada, troncos y hojas). Las paleocorrientes dominantes son hacia el NE-NW. Al igual que los depósitos de fangolitas de prodelta distal, estos presentan niveles con grietas de sinéresis. El contenido icnológico está representado por ejemplares de Teichichnus y Planolites en las fracciones más fangosas, mientras que Gyrochorte (Fig. 3F), y en menor medida Skolithos, han sido reconocidos al techo de los niveles arenosos que muestran óndulas simétricas.

Interpretación: el dominio de niveles de areniscas sobre las fangolitas en estos depósitos indica que los procesos tractivos asociados a flujos de baja energía fueron dominantes sobre los procesos de decantación. La presencia de delgados niveles de fango y materia orgánica en las caras de avalancha de las estructuras tractivas indica que la generación de flóculos, y su posterior transporte como carga de lecho, fue uno de los procesos y mecanismos de transporte más frecuente en el desarrollo de esta facies (Schieber $e t$ $a l ., 2007$; Ponce y Carmona, 2011). Al igual que en los depósitos de prodelta distal, el empobrecimiento que muestra el contenido icnológico, y la presencia de grietas de sinéresis, reflejan condiciones de estrés ambiental, posiblemente vinculadas con variaciones de salinidad y turbidez del agua (MacEachern et al., 2005). La presencia de Skolithos en niveles con óndulas simétricas sugiere que durante las etapas de retrabajo por oleaje se generaron ventanas de colonización para el establecimiento de organismos suspensívoros.

\subsection{Asociaciones de facies de frente deltaico distal y proximal}

Estas asociaciones de facies son dominantes en el tramo superior de la sección expuesta en Portada Covunco y en la Sierra de la Vaca Muerta, donde alcanzan un espesor máximo de $500 \mathrm{~m}$ (Figs. 1 y 2). En general, los depósitos muestran un arreglo granoestrato creciente integrado por areniscas finas a medianas, que pasan en transición hacia areniscas gruesas y a conglomerados finos acumulados en cuerpos de geometría tabular, que de manera subordinada presentan cuerpos lenticulares (Fig. 4A).

\subsubsection{Asociación de facies de frente deltaico distal}

Se halla integrada por areniscas finas a gruesas y, en menor medida, conglomerados finos y fangolitas dispuestos en cuerpos de geometría tabular de hasta 

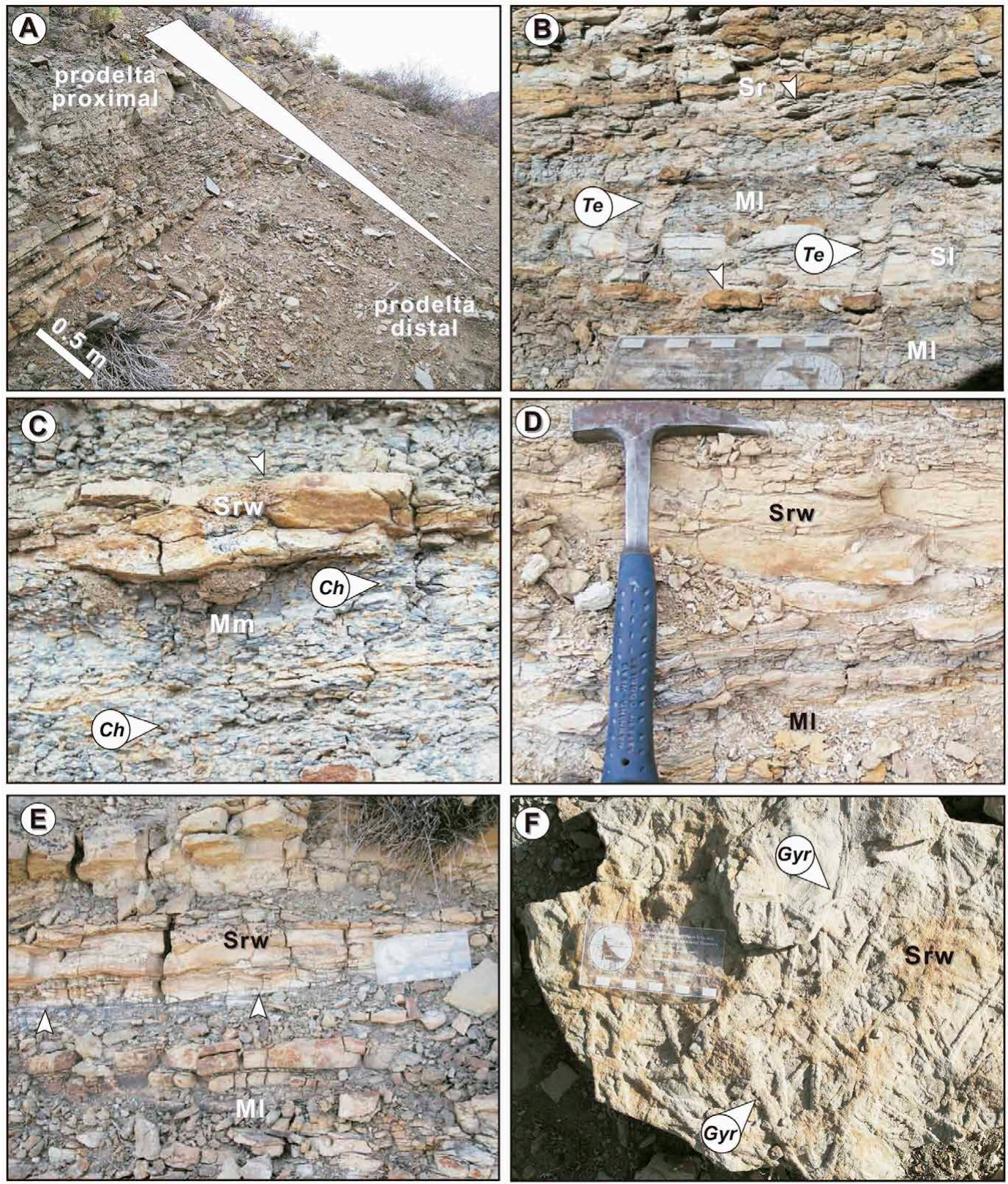

FIG. 3. Asociaciones de facies de prodelta. A. Arreglo grano-estrato creciente (barra blanca) donde se observa el pasaje transicional entre facies de prodelta distal y prodelta proximal; B-C. Heterolitas fangosas acumuladas en el prodelta distal, con Teichich$n u s$ ( Te) y Chondrites (Ch), y afectadas por acción de oleaje (flechas blancas); D-E. Heterolitas arenosas acumuladas en un prodelta proximal afectado por acción de oleaje (flechas blancas); F. Vista en planta de niveles de areniscas con Gyrochorte (Gyr). Mm: fangolitas macizas; Ml: fangolitas con laminación paralela; SI: areniscas con laminación paralela; Sr: areniscas finas con laminación ondulítica y lenticular; Srw: areniscas finas con laminación ondulítica simétrica.

1,5 $\mathrm{m}$ de espesor (Fig. 4A). En algunas ocasiones, estos cuerpos son truncados por sistemas de canales menores. Internamente los bancos tabulares pueden ser macizos o presentar estratificación entrecruzada tangencial y en artesa de mediana escala, laminación paralela y óndulas de corriente (Fig. 4B), que 

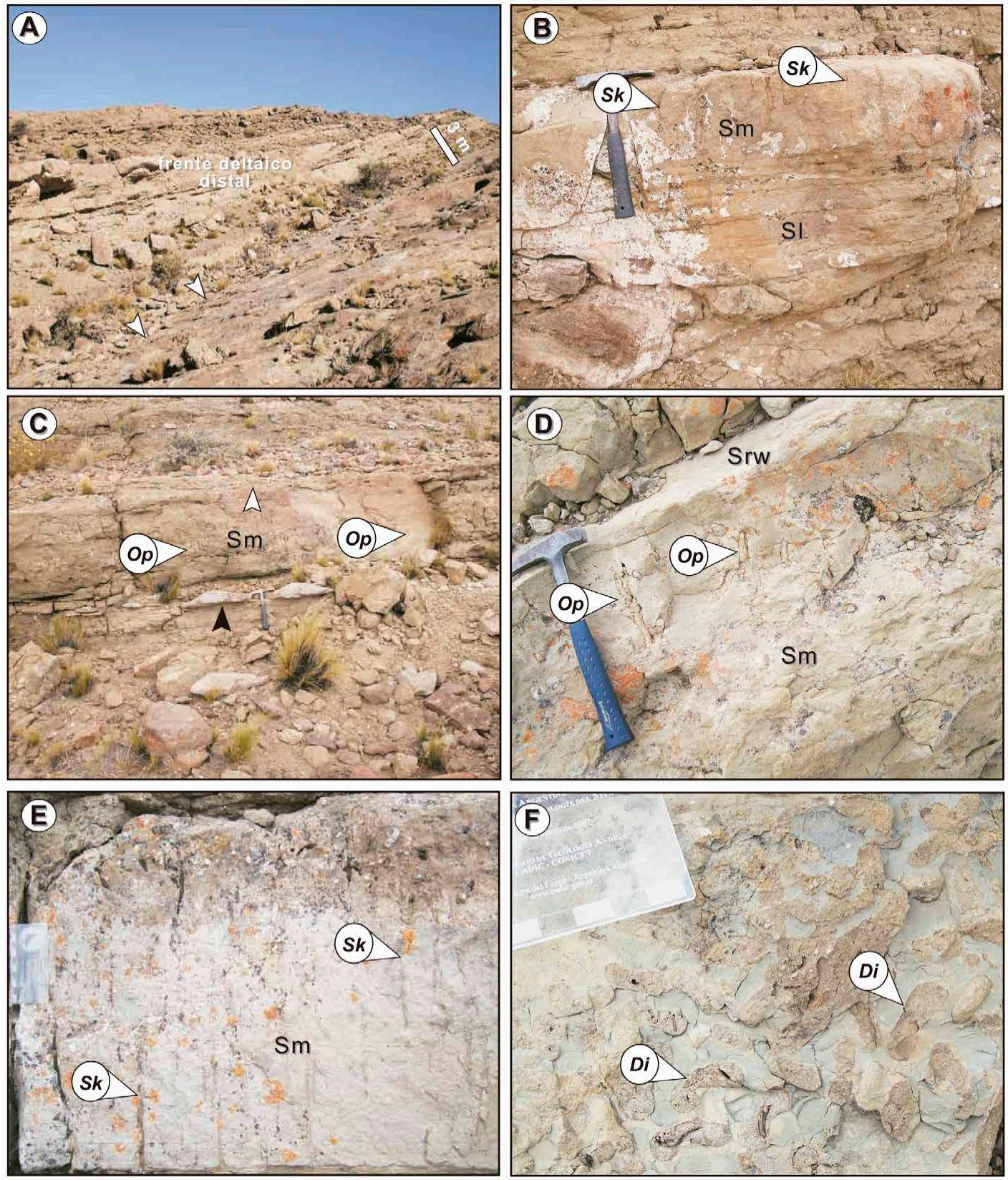

FIG. 4. Asociación de facies de frente deltaico distal. A. Vista general de niveles de arenas correspondientes a sistemas de lóbulos y barras acumuladas en un frente deltaico. Note la acción de oleaje al techo de los cuerpos tabulares (flechas blancas); B. Detalle de lóbulo de frente deltaico integrado por areniscas medias a finas con arreglos estrato-grano crecientes, retrabajadas por oleaje. Note hacia el techo de los depósitos ejemplares de Skolithos (Sk); C. Areniscas macizas completamente obliteradas por trazas fósiles asignadas a Ophiomorpha $(O p)$. Note la presencia de un nivel de megaóndulas generado por un evento de tormenta (flecha negra), y el retrabajo por acción de oleaje al techo del banco (flecha blanca); D. Vista en sección de areniscas con ejemplares de Ophiomorpha $(O p)$ y acción de oleaje al techo; E. Vista en sección de ejemplares de Skolithos (Sk) colonizando desde niveles de conglomerados con acción de oleaje al techo del depósito; F. Vista basal de una superficies sustrato-controlada (icnofacies de Glossifungites), integrada por estructuras biogénicas asignadas a Diplocraterion (Di) que presentan límites netos bien definidos y relleno pasivo. Sm: areniscas masivas; SI: areniscas con laminación paralela, Srw: areniscas finas con laminación ondulítica simétrica. 
incluyen intraclastos de fango, clastos alóctonos y bioclastos, así como también un abundante contenido de materia orgánica (particulada, troncos y hojas). Estos paquetes pueden estar completamente retrabajados por bioturbación, acción de oleaje, y/o presentar hacia el techo una acumulación de niveles de areniscas gruesas y conglomerados de hasta $0,4 \mathrm{~m}$ de espesor, con base erosiva y desarrollo de megaóndulas simétricas (flecha negra en Fig. 4C). Las paleocorrientes en estos depósitos indican una dirección de dispersión de sedimentos hacia el N-NE. El contenido icnológico está representado por Arenicolites, Gyrochorte, Ophiomorpha, Rhizocorallium, Skolithos y trazas de equilibrio/ escape de bivalvos (Fig. 4B-E). En algunos niveles se reconocieron superficies que no muestran gran continuidad lateral, con presencia de trazas fósiles con límites netos y morfología en $\mathrm{U}$, asignadas a Diplocraterion (Fig. 4F).

Interpretación: el dominio de niveles de areniscas finas a gruesas con desarrollo de estructuras tractivas que incluyen de manera subordinada conglomerados finos, y la escasa presencia de fangolitas, indican que los procesos tractivos asociados a flujos de alta energía fueron dominantes sobre los procesos de decantación. La abundante presencia de materia orgánica en los planos de estratificación de las estructuras tractivas sugiere una conexión directa con sistemas fluviales activos. El dominio de cuerpos tabulares, en ocasiones truncados por pequeños cuerpos lenticulares, se interpreta como sistemas de lóbulos generados por la coalescencia de barras de desembocadura deltaica, que en algunos casos se encuentran truncadas por canales distributarios terminales (Olariu y Bhattacharya, 2006). El contenido icnológico muestra una alta diversidad y abundancia, siendo dominantes las estructuras biogénicas producidas por organismos suspensívoros. Los depósitos de conglomerados con base erosiva y desarrollo de megaóndulas coronando los cuerpos tabulares se interpretan como depósitos de tormenta, los cuales constituyen ventanas de colonización para el establecimiento de organismos suspensívoros como Ophiomorpha y Skolithos. La presencia de niveles sin continuidad lateral y con desarrollo de la icnofacies de Glossifungites, se relaciona con procesos autocíclicos del sistema, donde el desarrollo de superficies sustrato-controladas indica la erosión por avulsión de los lóbulos deltaicos.

\subsubsection{Asociación de facies de frente deltaico proximal}

Esta asociación se presenta mejor expuesta en los tramos superiores de las secciones estudiadas en la Sierra de la Vaca Muerta. Corresponde a depósitos integrados por areniscas medianas a gruesas y conglomerados finos, dispuestos en cuerpos de geometría lenticular y tabular de hasta $6 \mathrm{~m}$ de espesor. Internamente muestran estratificación entrecruzada tangencial (con óndulas de corriente que migran en dirección opuesta a la migración de sus caras de avalancha), y en artesa de gran escala (Fig. 5A-B). La presencia de materia orgánica particulada se restringe a algunos pocos intervalos estratigráficos, siendo más frecuente la presencia de bioclastos y fragmentos de troncos dispuestos de manera caótica, o siguiendo los planos de estratificación. Estos depósitos muestran un retrabajo parcial o total por acción de oleaje (normal y de tormenta), con desarrollo de óndulas y megaóndulas (Fig. 5C). Las paleocorrientes indican una dirección de dispersión de sedimentos hacia el NE-E. El contenido icnológico muestra moderada diversidad y abundancia, con especímenes de Ophiomorpha, Skolithos, Macaronichnus, Dactyloidites, Curvolithus y trazas de equilibrio/escape (Fig. 5 B, D-F).

Interpretación: La presencia de cuerpos tabulares y lenticulares integrados por areniscas medianas a gruesas y conglomerados finos con estratificación entrecruzada tangencial y en artesa de gran escala, permite interpretar esta asociación de facies como sistemas de canales distributarios rellenos por barras y lóbulos deltaicos. Olariu y Bhattacharya (2006) indican que el reconocimiento de un gran número de sistemas de canales distributarios terminales rellenos por barras de desembocadura es diagnóstico de sistemas deltaicos fluvio-dominados. La presencia de intervalos estratigráficos integrados por barras de desembocadura deltaica afectadas de manera diferencial por acción de oleaje (normal y de tormenta), se relaciona con variaciones en la magnitud de la descarga fluvial. Durante descargas fluviales de alta magnitud se acumularon espesas barras de desembocadura deltaica que muestran acción de oleaje solamente al techo de las geoformas, mientras que durante descargas fluviales de baja magnitud, las barras son menos espesas y se encuentran más afectadas por la acción del oleaje. Este proceso favorece la generación de ventanas de colonización que permiten el establecimiento y desarrollo de una icnofacies de Skolithos con mezcla de componentes de la icnofacies de Cruziana. 

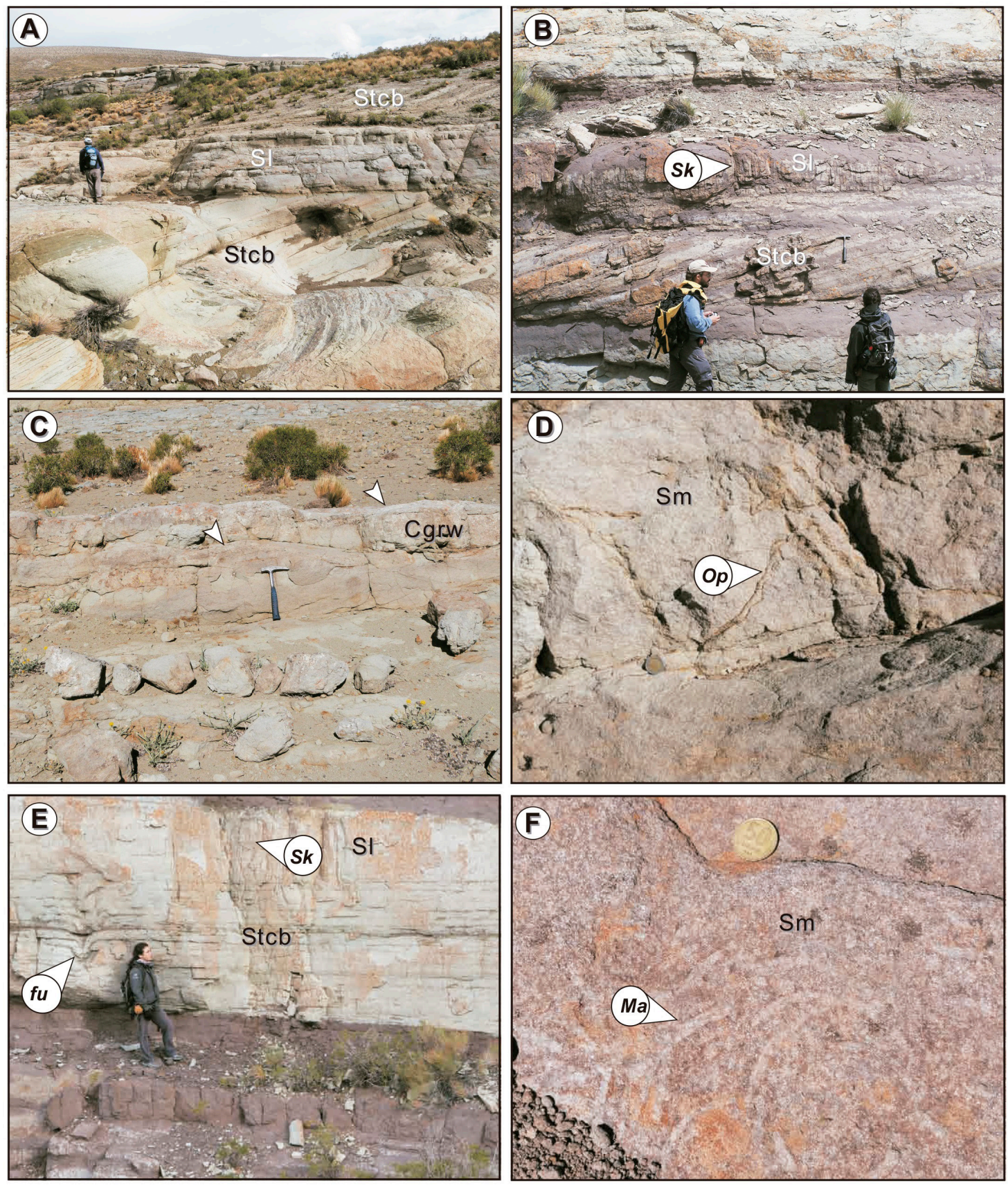

FIG. 5. Asociación de facies de frente deltaico proximal. A-B. Estratificación entrecruzada tangencial de gran escala y laminación paralela generada por la migración de barras de desembocadura deltaica. Note en B la presencia de Skolithos (Sk) en el topset de las barras; C. Areniscas medias a gruesas y conglomerados finos con desarrollo de megaóndulas (flechas blancas), vinculadas a procesos de tormenta que reelaboran el techo de las barras; D. Ejemplares de Ophiomorpha $(O p)$ en niveles de areniscas medias macizas y laminadas; E. Trazas de escape ( $f u)$ y ejemplares de Skolithos $(S k)$ en una barra de desembocadura deltaica; F. Vista en planta de ejemplares de Macaronichnus (Ma). Sm: areniscas macizas; Stcb: areniscas con estratificación entrecruzada tangencial y en artesa; SI: areniscas con laminación paralela; Cgrw: conglomerado fino con óndulas y megaóndulas. 


\subsection{Asociaciones de facies de canal y lóbulo hiperpícnico}

Los depósitos de prodelta y frente deltaico que integran las asociaciones de facies 1 y 2 descritas anteriormente, presentan intervalos estratigráficos compuestos por cuerpos lenticulares y tabulares producidos por descargas de densidad fluvio-derivadas (Fig. 2). Internamente estos depósitos difieren de los arreglos anteriormente mencionados, ya que muestran un pasaje transicional y recurrente entre diferentes estructuras sedimentarias con importantes variaciones texturales y múltiples superficies de reactivación interna (Zavala et al., 2006; Ponce y Carmona, 2011).

\subsubsection{Asociación de facies de canal hiperpícnico}

Esta asociación está compuesta por areniscas finas a medianas y, en menor medida, por conglomerados, acumulados en cuerpos de geometría lenticular con diferentes tamaños y tipos de relleno. Los cuerpos lenticulares mayores promedian los $8 \mathrm{~m}$ de espesor y $300 \mathrm{~m}$ de ancho, y solo han sido reconocidos en el tramo basal de la unidad, donde se encuentran truncando depósitos de prodelta proximal (Fig. 6). Internamente, muestran un pasaje transicional y recurrente de estructuras sedimentarias con marcadas variaciones texturales y múltiples superficies de reactivación. Los arreglos generalmente comienzan con areniscas finas macizas o con laminación paralela y dunas escalantes, que pasan hacia areniscas medianas a finas con estratificación en artesa y entrecruzada tangencial de gran escala, con múltiples superficies de reactivación (sri) (Figs. 6 y 7A-C). Estos paquetes incluyen clastos alóctonos, hojas, fragmentos de troncos y materia orgánica particulada, caóticamente dispersos en bancos de areniscas macizas (Fig. 7D), o dispuestos en los planos de estratificación de estructuras tractivas. En otros casos, los canales se encuentran rellenos por arenas macizas con estructuras de deformación por carga.

Los cuerpos lenticulares menores tienen $1 \mathrm{~m}$ de espesor y hasta $80 \mathrm{~m}$ de extensión lateral, poseen estructuras de acreción lateral y han sido principalmente reconocidos en las asociaciones de facies de frente deltaico distal y prodelta distal. Estos cuerpos conforman bancos de areniscas medianas a gruesas de hasta $0,5 \mathrm{~m}$ de espesor, con marcada geometría sigmoidal y planos que inclinan en dirección perpendicular a la corriente dominante (Fig. 7E). Internamente estos bancos presentan estructuras con láminación paralela y estratificación entrecruzada tangencial, con clastos alóctonos, fragmentos de troncos y fitodetrito en sus láminas. Las paleocorrientes indican una dispersión de sedimentos hacia el N-NE. Hacia el techo, y de manera recurrente, se reconocen niveles de hasta $50 \mathrm{~cm}$ de espesor integrados por conglomerados finos con óndulas y megaóndulas simétricas que muestran paleocorrientes dominantes del NW-SE y bases erosivas. Estos depósitos muestran baja diversidad y abundancia de trazas fósiles, con especímenes de Ophiomorpha, Skolithos y Arenicolites reconocidos al techo de los cuerpos lenticulares, y asociados a los niveles de conglomerados (Fig. 7F-G).

Interpretación: El pasaje transicional y la recurrencia que muestran las diferentes estructuras sedimentarias que integran estos depósitos, sus variaciones texturales, contenido de materia orgánica y geometría de los cuerpos permite interpretar a estos depósitos como sistemas de canales producidos por corrientes de densidad fluvio-derivadas (hiperpícnicas). En este contexto, la incisión principal del canal ocurre durante la fase de máxima aceleración de la descarga hiperpícnica, mientras que su relleno se produce durante la subsiguiente fase de desaceleración (Petter y Steel, 2006; Zavala et al., 2006; Ponce et al., 2007). Durante la fase de desaceleración, las variaciones en la velocidad (concentración) que experimenta el flujo dan lugar a la alternancia de estructuras tractivas de diferente escala, que en algunos casos pueden estar separadas por superficies de reactivación (sri en Figs. 6 y 7A-B). La presencia de clastos alóctonos y fragmentos de troncos con sus ejes mayores dispuestos de manera perpendicular a la dirección de la corriente, así como la presencia de fragmentos de hojas dispuestas de manera caótica dentro de las capas, sugiere que la combinación entre carga de lecho y suspensión turbulenta fue muy importante durante la sedimentación. Los canales mayores cuyos rellenos están integrados por arenas macizas con estructuras de deformación por carga, se habrían acumulado en zonas de cambio de pendiente, donde las corrientes hiperpícnicas experimentan una rápida agradación por expansión y pérdida de parte de la carga transportada en suspensión turbulenta (Macdonald et al., 2009; Ponce y Carmona, 2011). De acuerdo a sus características internas, y a la relación que muestran con las asociaciones de facies 

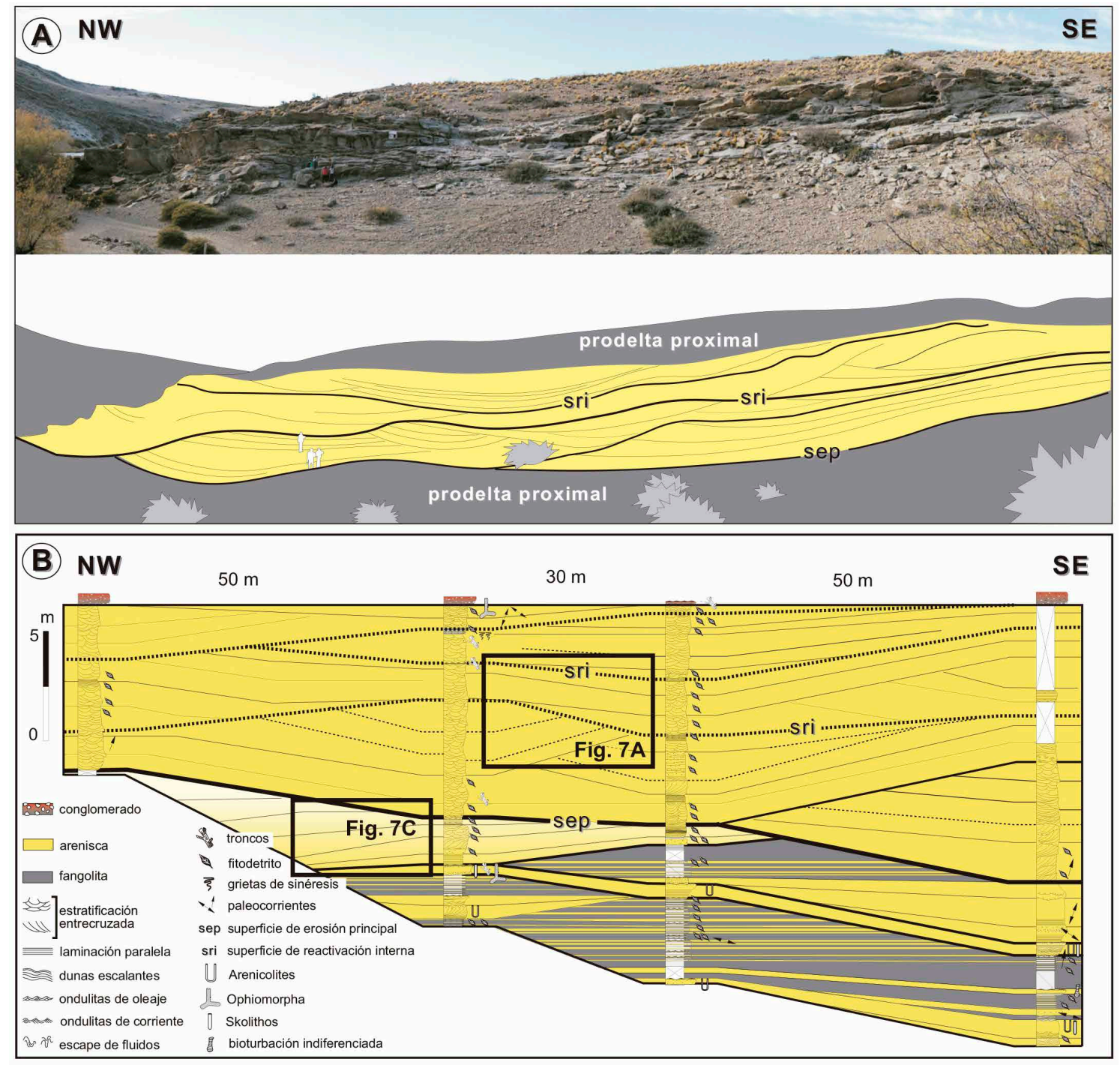

FIG. 6. Asociación de facies de canal hiperpícnico. A. Vista panorámica y redibujo de un canal hiperpícnico mayor truncando depósitos de prodelta proximal. En estos cuerpos domina el relleno agradacional; B. Panel de correlación del canal hiperpícnico mostrado en $\mathbf{A}$, donde se indica la ubicación de la superficie erosiva principal (sep), y las múltiples superficies de reactivación interna (sri).

de prodelta y frente deltaico, se pudo determinar que los canales mayores se originan al pie de las zonas de mayor gradiente, donde el poder erosivo de los flujos se ve incrementado, mientras que los canales menores de alta sinuosidad se desarrollan en zonas de menor gradiente, donde el poder erosivo de la corriente disminuye de manera considerable.

Los conglomerados finos con óndulas y megaóndulas simétricas y abundante contenido de materia orgánica que se encuentran coronando los arreglos de los canales principales, indican que la acción de oleaje (normal y de tormenta) fue el proceso sobrepuesto dominante para la conformación de estos depósitos (Fig. 7F-G). El continuo retrabajo por oleaje permite que las arenas escapen del sistema, generando una superficie de 'lavado' donde se produce la concentración de sedimentos gruesos. Estas superficies marcan planos de pausa en la sedimentación hiperpícnica, permitiendo el establecimiento de ventanas de colonización para la fauna bentónica. 

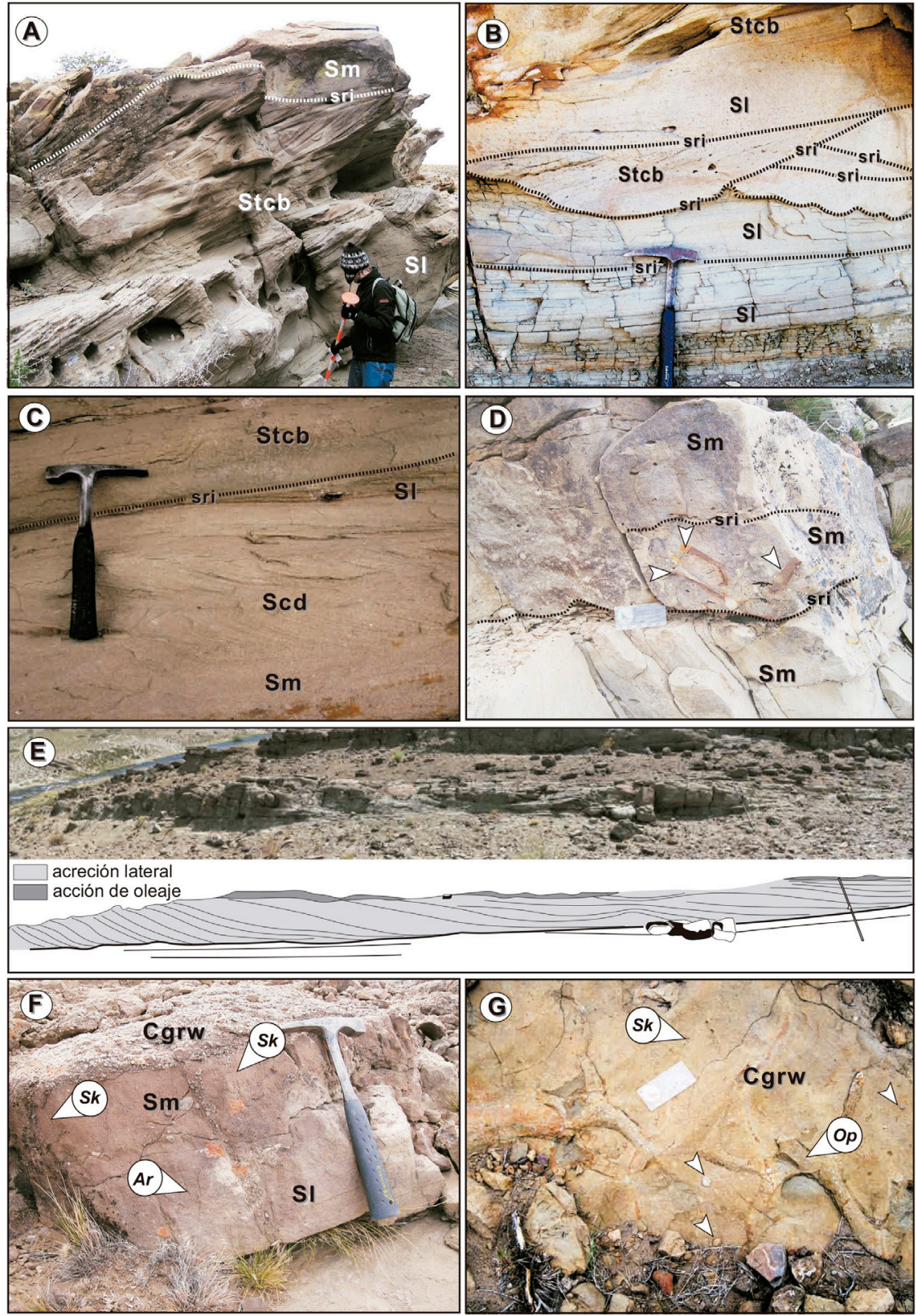

FIG. 7. Asociación de facies de canal hiperpícnico. A. Areniscas medias a finas y conglomerados finos acumulados en sistemas de canales hiperpícnicos mayores con relleno agradacional. Note el pasaje transicional entre estructuras tractivas y macizas, que en algunos casos se encuentran separadas por superficies de reactivación interna; B-D. Pasaje transicional y recurrente entre diferentes estructuras sedimentarias producidas por variaciones de concentración y velocidad del flujo en el relleno de los canales mayores. Note en $\mathbf{D}$, la presencia de hojas y troncos (flechas blancas) dispuestos de manera caótica dentro de niveles de arena masiva; E. Imagen y redibujo de un canal hiperpícnico menor con desarrollo de estructuras de acreción lateral y acción de oleaje hacia el techo; F. Vista en sección de ejemplares de Skolithos (Sk) y Arenicolites (Ar) colonizando niveles de conglomerados finos afectados por acción de oleaje al techo de los sistemas de canales; G. Vista en planta de ejemplares de Ophiomorpha $(O p)$ y Skolithos (Sk) preservados en niveles de areniscas gruesas con óndulas simétricas y clastos alóctonos (flechas blancas). Sm: areniscas macizas; Stcb: areniscas con estratificación entrecruzada tangencial y en artesa; Sl: areniscas con laminación paralela; Scd: areniscas con dunas escalantes; Cgrw: conglomerado fino con óndulas y megaóndulas; sri: superficies de reactivación interna. 


\subsubsection{Asociación de facies de lóbulo hiperpícnico}

Estos depósitos se encuentran dominados por cuerpos de geometría tabular de gran extensión lateral y espesor variable (Fig. 8 A-B), que muestran una dispersión de sedimentos dominante hacia el NW. Internamente, los paquetes tienen un arreglo grano-estrato creciente sucedido por uno decreciente, comenzando con sucesiones de heterolitas de hasta $1 \mathrm{~m}$ de espesor, integradas por una alternancia centimétrica de fangolitas laminadas y macizas, con abundante contenido de materia orgánica y estructuras de deformación, y areniscas macizas o con laminación paralela y óndulas de corriente, con abundante contenido de fangolitas y materia orgánica en sus caras de avalancha. Estos depósitos son sucedidos en transición por areniscas finas a medianas macizas, que pasan hacia conglomerados integrados por clastos alóctonos de hasta $4 \mathrm{~cm}$ de longitud, dispuestos sobre superficies de reactivación (sri), o alineados a planos de estratificación difusos, culminando con depósitos de arenas medianas a finas macizas o con laminación paralela (Fig. 8C-D). En ocasiones se puede reconocer que los cuerpos tabulares presentan un arreglo grano-estrato decreciente, donde areniscas gruesas a conglomerádicas dispuestas sobre superficies erosivas muestran un pasaje transicional hacia areniscas macizas o con laminación paralela y estratificación entrecruzada tangencial, culminando con areniscas medias a finas macizas o con laminación paralela (Fig. 8E). Hacia el techo de estos cuerpos se reconocen depósitos de areniscas gruesas o conglomerados con óndulas y megaóndulas simétricas de hasta $30 \mathrm{~cm}$ de espesor y base erosiva (Fig. 8C, F). El contenido icnológico muestra una diversidad levemente mayor que la observada en los canales hiperpícnicos, con ejemplares de Skolithos, Arenicolites, Ophiomorpha, y Gyrochorte, así como trazas de escape (fugichnia) (Fig. 8C, F-G).

Interpretación: La geometría tabular que muestran los depósitos y el pasaje transicional y recurrente de las estructuras sedimentarias permiten interpretar estos arreglos como lóbulos hiperpícnicos. Los arreglos grano y estrato creciente-decreciente representan la acumulación generada durante un ciclo completo de descarga hiperpícnica (aceleración-máxima eficiencia-desaceleración; ver también Zavala et al., 2006), mientras que los paquetes grano y estrato decrecientes representan la acumulación producida durante las fases de máxima eficiencia -desaceleración del flujo. En este último caso, la no preservación de las sucesiones acumuladas durante la fase de aceleración se debe al desarrollo prolongado de la fase de máxima eficiencia, donde los procesos erosivos son dominantes (Zavala et al., 2006). La presencia de óndulas y megaóndulas simétricas acumuladas sobre superficies erosivas que se encuentran coronando los arreglos indica que la acción de oleaje fue el proceso dominante sobrepuesto en estos depósitos. $\mathrm{Al}$ igual que en los canales hiperpícnicos, el reconocimiento de estos depósitos permite establecer los planos de pausa en las descargas hiperpícnicas y los momentos en los cuales se producen las ventanas de colonización de organismos suspensívoros (e.g., Arenicolites, Ophiomorpha, Skolithos).

\section{Discusión}

\subsection{Origen de los canales en la sucesión deltaica}

La Formación Lajas en las localidades de Portada Covunco y Sierra de la Vaca Muerta conforma una sucesión general grano-estrato creciente donde depósitos de fangolitas y heterolitas correspondientes a facies de prodelta son reemplazados de manera transicional por areniscas y conglomerados acumulados en un frente deltaico (Fig. 2). Un aspecto sumamente interesante de esta sucesión es la presencia de canales mayores con relleno agradacional restringidos a las facies de prodelta proximal (Fig. 6) y de canales menores con estructuras de acreción lateral restringidos a las facies de frente deltaico y prodelta distal (Fig. 7E). Estos canales constituyen un rasgo anómalo en la evolución del arreglo progradante que caracteriza a los sistemas deltaicos. La presencia de sistemas de canales en facies de prodelta ha sido relacionada con la generación de corrientes de densidad producidas por desestabilizaciones de un frente deltaico o por el desarrollo de descargas hiperpícnicas (Nemec, 1995; Mulder y Syvitski, 1996). Kineke et al. (2000) documentan la presencia de ambos procesos en la desembocadura del río Sepik en Papúa, Nueva Guinea. Estas observaciones sugieren que la alternancia entre condiciones de descargas hipopícnicas e hiperpícnicas en los sistemas deltaicos son sumamente frecuentes (Nemec, 1995; Mulder and Syvitski, 1995), principalmente en aquellos deltas de tipo fluvio-dominados. Los depósitos producidos por plumas hiperpícnicas generalmente muestran arreglos 

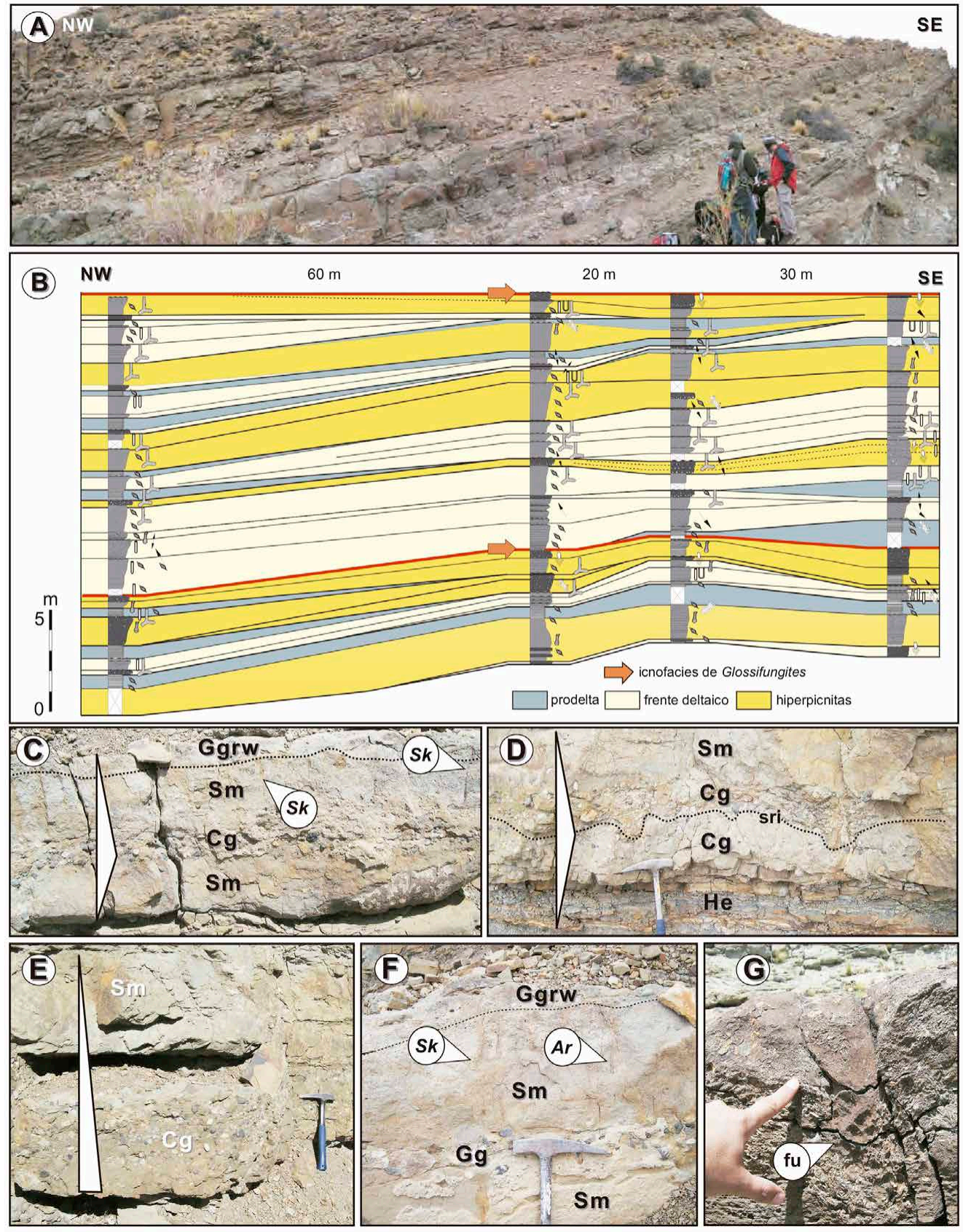

FIG. 8. Asociación de facies de lóbulo hiperpícnico. A-B. Vista panorámica y panel de correlación mostrando los intervalos estratigráficos integrados por lóbulos y canales de alta sinuosidad hiperpícnicos en la sucesión deltaica; C-D. Cuerpos tabulares con arreglos grano-estrato creciente sucedidos por uno grano-estrato decreciente, producidos durante un ciclo completo de descarga hiperpícnica (aceleración-máxima eficiencia-desaceleración), que internamente muestran pasajes transicionales entre diferentes estructuras sedimentarias con múltiples superficies de reactivación interna; E. Arreglos de tipo grano-estrato decreciente producido por la canibalización total de los depósitos acumulados durante la etapa de aceleración, debido al desarrollo de una zlif erosiva; F. Cuerpos tabulares con arreglos grano-estrato creciente sucedidos por uno grano-estrato decreciente y acción de oleaje al techo, bioturbados por Skolithos (Sk) y Arenicolites (Ar); G. Vista en sección de trazas de escape de bivalvos (fu). Cg: conglomerados; Cgrw: conglomerados con óndulas simétricas; Sm: areniscas masivas; He: heterolitas; sri: superficies de reactivación interna. 
más complejos que los producidos por corrientes de densidad generadas por desestabilizaciones en el frente deltaico, debido a la mayor duración y a las fluctuaciones de velocidad y concentración de sedimentos que experimenta el flujo (Ponce et al., 2007). Las observaciones realizadas en los depósitos de la Formación Lajas indican la existencia de una alternancia entre depósitos acumulados por plumas hipopícnicas e hiperpícnicas. Esta alternancia en el tipo de descarga fluvial produce cuerpos cuyas geometrías, asociaciones de facies sedimentarias y contenido icnológico resultan propias y son características para cada proceso de sedimentación. Las diferencias observadas en las dimensiones de los canales hiperpícnicos responden principalmente a un control en el gradiente del sistema. De este modo, al pie de las zonas de mayor gradiente, el poder erosivo de la corriente hiperpícnica se incrementa produciendo las mayores incisiones, mientras que en las zonas de menor gradiente, los sistemas de canales hiperpícnicos presentan dimensiones menores y un trazado de alta sinuosidad como lo sugiere la presencia de estructuras de acreción lateral. Un caso similar fue documentado por Ponce y Carmona (2011) en sistemas de canales hiperpícnicos desarrollados al pie de clinoformas progradantes en ambientes marinos profundos del Mioceno de Cuenca Austral, Argentina.

\subsection{Carácter fluvio-dominado de los depósitos}

Dependiendo de los procesos dominantes, los sistemas deltaicos desarrollan morfologías distintivas que permiten clasificarlos como fluvio-dominados, dominados por olas o dominados por mareas (Galloway, 1975). Domínguez (1996) y Bhattacharya (2006) sugieren que esta clasificación debe ser usada con precaución, debido a que la configuración de los sistemas deltaicos puede variar en el tiempo y el espacio como resultado de la combinación de más de un proceso de sedimentación activo. Por otra parte, y como resultado de efectos climático-estacionales, los ríos experimentan cambios en la concentración de sedimentos transportados durante su descarga (Bhattacharya, 2006). Como consecuencia de esto, muchos sistemas fluviales alternan entre condiciones de descargas hipopícnicas e hiperpícnicas (Nemec, 1995; Mulder y Syvitski, 1995; Parsons et al., 2001). Durante las descargas fluvio-derivadas (normales y extraordinarias) se producen impor- tantes modificaciones de las propiedades físicas y químicas en la columna de agua (e.g., salinidad, oxigenación, temperatura, turbidez), que afectan el desarrollo de las comunidades bentónicas. En este sentido, estudios icnológicos en sucesiones deltaicas (MacEachern et al., 2005) e hiperpícnicas de ambientes marinos someros (Saccavino et al., 2008; Buatois et al., 2011) y profundos (Wetzel, 2008; Carmona y Ponce, 2011) mostraron que las descargas fluvio-derivadas ejercen un control muy importante en la diversidad y distribución de las estructuras biogénicas. Estos conceptos indican que la caracterización de ambientes deltaicos e hiperpícnicos sobre la base del análisis de facies, geometría de los cuerpos y contenido icnológico puede ser en muchos casos sumamente compleja, debido a la interacción que se produce entre los diferentes procesos de sedimentación.

Los depósitos de la Formación Lajas en las áreas de Portada Covunco y Sierra de la Vaca Muerta han sido interpretados como depósitos deltaicos con descargas hiperpícnicas, afectados por olas y mareas (Zavala y González, 2001), y como sistemas deltaicos dominados por olas y tormentas (McIlroy et al., 2005). Si bien la acción de oleaje constituye el proceso de retrabajo principal reconocido en estos depósitos, la presencia de estructuras físicas consideradas diagnósticas de procesos mareales, tales como laminación flaser, herringbone, tidal bundles, estructuras bidireccionales tapizadas por cortinas de fango (Davis, 2012) y tidalitas tubulares (Gingras et al., 2007; Wetzel et al., 2014), no han sido aún reconocidas. Por el contrario, estos depósitos se encuentran dominados por estructuras sedimentarias unidireccionales con abundante contenido de materia orgánica, acumulada en las caras de avalancha de estructuras tractivas de diferente escala. Este tipo de arreglo ha sido reconocido en depósitos de lóbulos y albardón hiperpícnicos marinos profundos del Mioceno de Cuenca Austral (Ponce, 2009; Ponce y Carmona, 2011), e interpretado como el producto de procesos de floculación, donde los agregados, hidráulicamente equivalentes a arenas finas, son transportados como carga de lecho y acumulados en las caras de avalancha de las estructuras tractivas durante su migración, generando arreglos similares a los que presentan las estructuras de tidal bundle y estratificación flaser. A diferencia de las estructuras mareales donde las cortinas de fango se producen por decantación durante períodos de baja energía de la 
marea (slack water), la presencia de fangos y materia orgánica, conjuntamente con granos de areniscas, en la gran mayoría de las caras de avalancha de las estructuras tractivas reconocidas en la Formación Lajas, indica que su acumulación fue producida durante la migración de las estructuras sedimentarias. El reconocimiento de este tipo de estructuras, sumado a la presencia de materia orgánica detrítica (fragmentos de hojas y troncos), distribuida de manera caótica en niveles de areniscas macizas, indican una directa vinculación con descargas fluvio-derivadas (normales y de crecida), más que con procesos de difusión mareal activos para estos depósitos (Ponce y Carmona, 2011; Zavala et al., 2012). Por otra parte, no se ha reconocido la presencia de sistemas de galerías con relleno mareal, denominadas tidalitas tubulares (Gingras et al., 2007; Wetzel et al., 2013, 2014); por el contrario, la gran mayoría de las excavaciones asignadas a Ophiomorpha muestran rellenos arenosos, con abundante contenido de materia orgánica y escasa presencia de niveles de fango. Del mismo modo, el reconocimiento de sistemas de barras de desembocadura deltaica rellenando numerosos sistemas de canales distributarios terminales al techo de los depósitos de la Formación Lajas en la Sierra de la Vaca Muerta, es un rasgo característico de sistemas deltaicos fluvio-dominados (Olariu y Bhattacharya, 2006). Por lo expuesto anteriormente, estos sistemas deltaicos corresponderían preferentemente a los de tipo fluvio-dominados y no a deltas dominados por marea.

\subsection{Contenido icnológico}

Los depósitos analizados muestran diferentes asociaciones icnológicas en cada una de sus asociaciones de facies. Los depósitos de prodelta se caracterizan por la presencia de una suite de organismos depositívoros, característicos de una icnofacies de Cruziana empobrecida, con algunas trazas asignadas a organismos suspensívoros en niveles arenosos con acción de oleaje. Los depósitos de frente deltaico presentan suites de trazas fósiles con dominio de estructuras de organismos suspensívoros, característicos de la icnofacies de Skolithos. Esta suite es la que presenta la mayor diversidad y abundancia de estructuras biogénicas en el sistema. Por otro lado, los depósitos hiperpícnicos muestran dominio de estructuras de organismos suspensívoros, con suites de moderada a baja diversidad. Si bien los sistemas de lóbulos hiperpícnicos tienen una diversidad levemente mayor que los sistemas de canales hiperpícnicos, en general estos depósitos muestran asociaciones de trazas fósiles más empobrecidas que las reconocidas en las facies deltaicas, con poco desarrollo de la estructura de escalonamiento y dominio de estrategias oportunistas.

Los principales factores de estrés ambiental que afectaron a las comunidades bentónicas fueron las fluctuaciones en la salinidad y en la energía y las altas tasas de sedimentación generadas durante la descarga de los flujos fluvio-derivados. La presencia de niveles con grietas de sinéresis, principalmente al techo de niveles de canales hiperpícnicos, evidencia la existencia de marcadas fluctuaciones de salinidad en el ambiente. Con seguridad, la reducción en la salinidad provocada por la entrada de agua dulce aportada durante la descarga hiperpícnica tuvo mayor efecto en los sectores proximales del sistema que en los distales. El desarrollo de asociaciones icnológicas de baja diversidad, o la ausencia de las mismas en los sistemas de canales hiperpícnicos, sustenta la idea de que en los ambientes proximales se dieron condiciones de mayor estrés salino y energético, mientras que en las posiciones distales del sistema, con desarrollo de lóbulos hiperpícnicos, se presenta la mayor diversidad icnológica. Estas características icnológicas fueron también reconocidas por Carmona y Ponce (2011) en sucesiones hiperpícnicas marinas profundas del Mioceno de la Cuenca Austral.

El reconocimiento de superficies sustratocontroladas representadas por la icnofacies de Glossifungites en los sistemas de lóbulos de frente deltaico es de gran importancia estratigráfica, ya que permite reconocer superficies exhumadas generadas por la avulsión de los sistemas de lóbulos y su posterior colonización. Finalmente, la presencia de los niveles de conglomerados con retrabajo por acción del oleaje refleja la presencia de intervalos de pausa en la sedimentación, con desarrollo de ventanas de colonización en las que se establecieron organismos suspensívoros (e.g., Ophiomorpha, Skolithos, Arenicolites).

\subsection{Arreglo interno de los depósitos hiperpícnicos}

En la zona bajo estudio, las hiperpicnitas que integran los depósitos de la Formación Lajas muestran frecuentemente un pasaje transicional y recurrente entre diferentes estructuras sedimentarias 
que incluyen variaciones texturales, superficies de reactivación, arreglos grano-crecientes sucedidos por arreglos grano-decrecientes, y una gran concentración de materia orgánica distribuida caóticamente en arenas macizas, o dispuestas sobre los planos de estratificación de diversas estructuras tractivas. Un concepto clave para comprender los procesos y mecanismos de sedimentación que dan lugar a este tipo de arreglos es el de zona de límite inferior de flujo (zlif), definida por Branney y Kokelaar (1992, 2002). Dichos autores aplican este concepto para explicar la transición entre estructuras sedimentarias y las variaciones texturales reconocidas en depósitos generados por corrientes de densidad sostenidas de origen piroclástico. La zlif se define como la porción más baja de la corriente de densidad y la parte más alta del depósito en formación (Fig. 9A), la cual varía progresivamente en la medida en que el depósito agrada o es erosionado bajo condiciones de flujo sostenido. Las variaciones en la naturaleza de este límite dependen de la velocidad de la corriente, de la concentración de partículas y de la duración del evento de sedimentación. De este modo, cuando la concentración de partículas en la parte superior del depósito en formación es mucho mayor que en la parte inferior del flujo sobrepasante, se produce un contraste reológico que posibilita que los clastos de diferente tamaño se muevan libremente como carga de lecho sobre esta superficie, generando, de acuerdo a las fluctuaciones de velocidad del flujo, un pasaje transicional y recurrente entre diferentes estructuras tractivas (Fig. 9B). Por el contrario, cuando la concentración de clastos al tope del depósito en formación y en la porción basal de la corriente de densidad es muy alta, no existe un contraste reológico entre el depósito y el flujo en
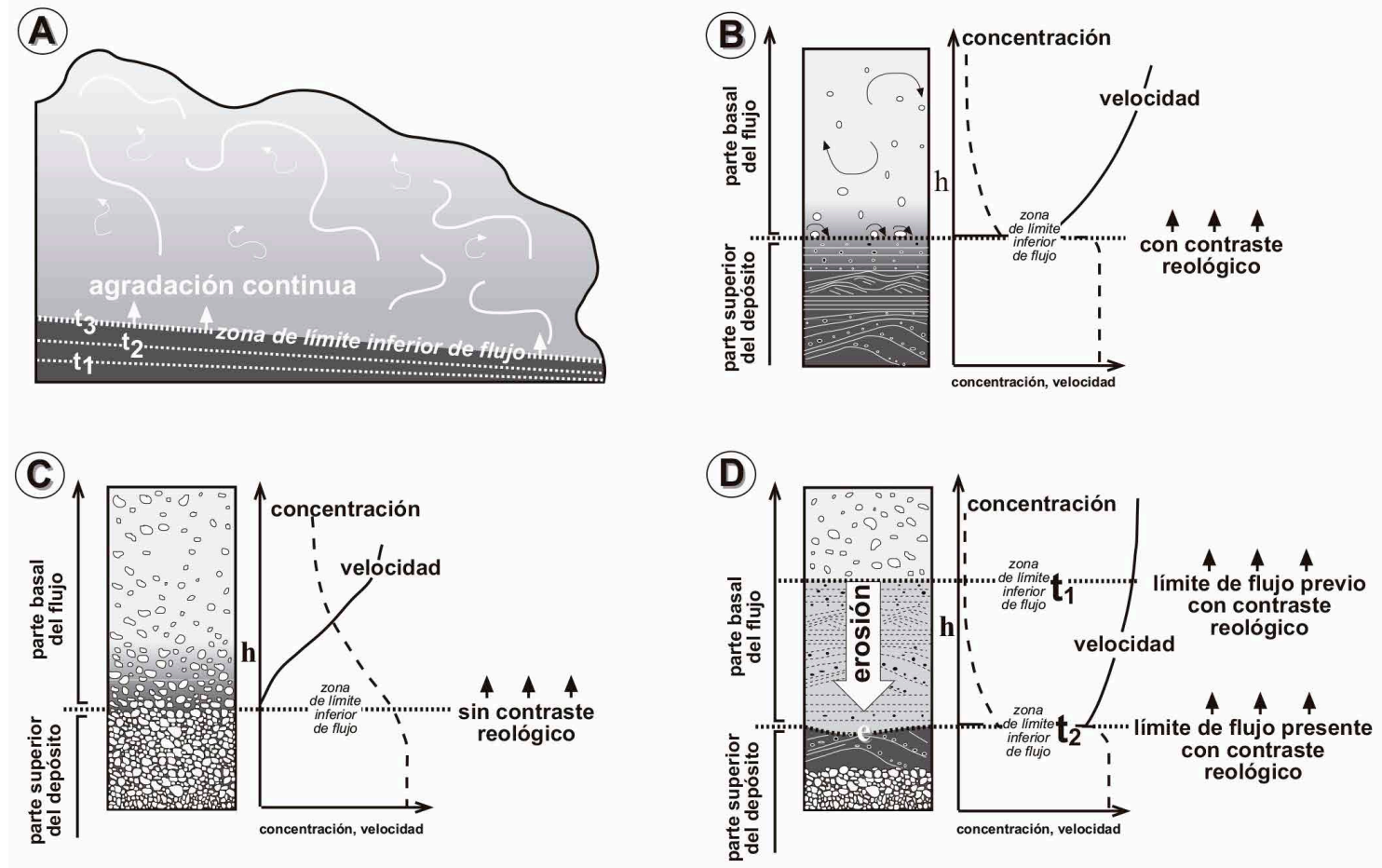

FIG. 9. A. Esquema de una corriente de densidad sostenida donde se indica la migración progresiva de la zona de límite inferior de flujo (zlif) por agradación durante el mismo evento de sedimentación $\left(\mathrm{t}_{1}-\mathrm{t}_{3}\right)$; B. Evolución de una zlif tractiva, caracterizada por presentar una alta concentración de partículas en la parte superior del depósito y una baja concentración en la parte inferior del flujo, favoreciendo el desarrollo de un pasaje transicional entre diferentes estructuras tractivas; C. Evolución de una zlif maciza, donde la alta concentración de sedimentos en la parte superior del depósito en formación e inferior de la corriente, favorecen la depositación por agradación de capas macizas de espesor variable; D. Evolución de una zlif erosiva, donde los depósitos previos son parcial o totalmente canibalizados por un flujo de densidad sostenido que supera el umbral de erosión de las fracciones previamente acumuladas (tomado y modificado de Branney y Kokelaar, 2002; Ponce, 2009; Ponce y Carmona, 2011). 
formación, produciéndose agradación continua con desarrollo de niveles macizos que pueden producir estructuras de deformación por carga (Fig. 9C). Cuando el flujo de densidad en aceleración supera el rango de velocidad necesaria para producir erosión, el depósito previamente acumulado puede ser parcial o totalmente canibalizado, dependiendo del tiempo durante el cual el flujo mantenga la velocidad que supere dicho umbral de erosión (Fig. 9D). El carácter intergradacional y recurrente del desarrollo de una zlif, así como sus variaciones espaciales y temporales, permiten explicar adecuadamente las características internas que muestran los depósitos de canal y lóbulo hiperpícnicos reconocidos en la Formación Lajas.

\subsection{Modelo depositacional}

Los depósitos de la Formación Lajas en las áreas de Portada Covunco y Sierra de la Vaca Muerta representan un sistema deltaico fluvio-dominado que se encuentra frecuentemente afectado por descargas hiperpícnicas, y reelaborado por acción de oleaje (Canale y Ponce, 2012; Ponce et al., 2012). El arreglo general grano-estrato creciente que muestra la sucesión analizada resulta de la progradación hacia el NW y NE de sucesiones de areniscas y conglomerados de frente deltaico sobre heterolitas de prodelta, acumuladas durante etapas de sedimentación normal en la cuenca (Figs. 2 y 10A). Durante estas etapas se producen los depósitos que muestran la mayor diversidad icnológica del sistema, principalmente en las asociaciones de facies de frente deltaico. Estos depósitos se encuentran truncados por sistemas de canales y lóbulos generados por corrientes de densidad hiperpícnicas (Figs. 2 y 10B). Al pie de las zonas de mayor gradiente, es decir, entre el frente deltaico distal y el prodelta proximal, se generaron los canales hiperpícnicos de mayores dimensiones con relleno agradacional (Fig. 10B), mientras que en las posiciones de menor gradiente del sistema se desarrollaron canales de alta sinuosidad (Fig. 10B). Los sistemas de lóbulos hiperpícnicos ocurren en zonas de bajo gradiente donde el flujo pierde confinamiento, y representan las posiciones más distales de los sistemas de descargas hiperpícnicas (Fig. 10B). Las hiperpicnitas presentan la más baja diversidad icnológica debido a que las condiciones de estrés, como son los cambios de salinidad, turbidez del agua, y principalmente, la tasa de sedimentación, son máximas. La presencia de numerosas superficies sustrato-controladas $\sin$ extensión regional, principalmente reconocidas en los depósitos de frente deltaico, se habrían generado por la avulsión de sistema de lóbulos (Fig. 10C). La presencia de cuerpos tabulares integrados por conglomerados y areniscas gruesas en la Sierra de la Vaca Muerta, sugiere que la migración del sistema fue hacia el W-NW (Fig. 10C). Durante la etapa final de la evolución, la progradación de los depósitos de frente deltaico proximal genera complejos de barras y canales distributarios terminales, y una dispersión de sedimentos dominante hacia el NE-E. Estos sistemas muestran una alternancia entre etapas de sedimentación normal y de crecida (Fig. 10D). Tanto los depósitos deltaicos como hiperpícnicos se encuentran reelaborados por acción de oleaje normal y de tormenta, constituyendo el proceso sobrepuesto dominante en este sistema. Depósitos similares integrados por deltas fluvio-dominados con descargas hiperpícnicas asociadas, han sido documentados sobre la base de datos de subsuelo para el Cretácico superior de la Formación Magallanes, Patagonia, Argentina (Buatois et al., 2011).

\section{Conclusiones}

- El análisis sedimentológico e icnológico de los depósitos de la Formación Lajas en las áreas de Portada Covunco y Sierra de la Vaca Muerta permitió reconocer facies sedimentarias típicas de ambientes deltaicos fluvio-dominados, frecuentemente afectados por descargas hiperpícnicas, y reelaborados por procesos de acción de oleaje (normal y de tormenta).

- La asociación de facies de prodelta se encuentra representada por cuerpos tabulares que muestran una alternancia centimétrica entre fangolitas macizas y/o laminadas, que de manera subordinada incluye areniscas finas con laminación ondulosa y lenticular, con abundante contenido de materia orgánica particulada. El contenido icnológico muestra intervalos no bioturbados o moderadamente bioturbados por organismos depositívoros, característicos de una icnofacies de Cruziana empobrecida.

- La asociación de facies de frente deltaico se encuentra integrada por areniscas finas a gruesas y en menor medida conglomerados finos y fangolitas dispuestas en cuerpos de geometría tabular, 


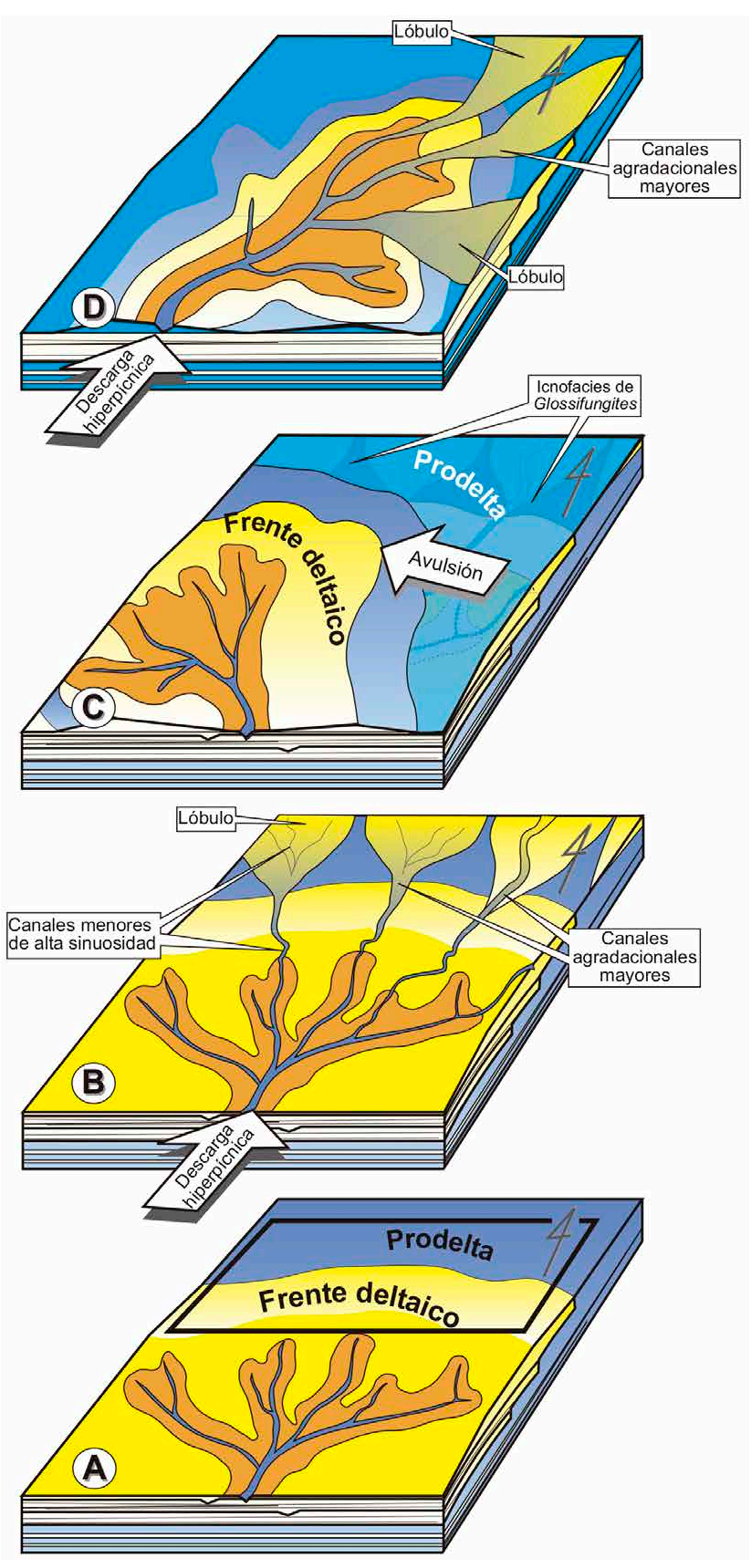

FIG. 10. Modelo paleoambiental para los depósitos de la Formación Lajas en las áreas de Portada Covunco y Sierra de la Vaca Muerta. A. Etapa de sedimentación normal con desarrollo de un arreglo general grano-estrato creciente que resulta de la progradación hacia el NW y NE de sucesiones de areniscas y conglomerados de frente deltaico sobre heterolitas de prodelta. El rectángulo indica la posición relativa que ocupan los depósitos analizados; B. Durante esta etapa, al pie de las zonas de mayor gradiente (e.g., prodelta proximal), el poder erosivo de la corriente hiperpícnica se incrementa produciendo las mayores incisiones, mientras que en las zonas de menor gradiente (e.g., frente deltaico y prodelta distal), los sistemas de canales hiperpícnicos presentan dimensiones menores y un trazado de alta sinuosidad. Los sistemas de lóbulos hiperpícnicos ocurren en zonas de bajo gradiente donde el flujo pierde confinamiento; C. Migración lateral del sistema por avulsión de lóbulos deltaicos con desarrollo de superficies sustrato-controladas (icnofacies de Glossifungites); D. Etapa con alternancia entre condiciones de sedimentación normal (depósitos de prodelta y frente deltaico) y descargas hiperpícnicas. 
que ocasionalmente son truncados por sistemas de canales menores. Estos depósitos corresponden a sistemas de barras y canales distributarios terminales. El contenido icnológico muestra una alta diversidad y abundancia, con predominio de estructuras biogénicas producidas por organismos suspensívoros (icnofacies de Skolithos). La presencia de niveles sin continuidad lateral con desarrollo de la icnofacies de Glossifungites, marca la presencia de superficies sustrato-controladas generadas por la avulsión de lóbulos deltaicos.

- La asociación de facies hiperpícnicas muestra dos arreglos característicos. Uno de ellos corresponde a sistemas de canales mayores con relleno agradacional y canales menores con estructuras de acreción lateral, cuyo desarrollo se encuentra controlado por el gradiente del sistema. El otro tipo de arreglo corresponde a cuerpos de geometría tabular, donde paquetes grano-estrato crecientes son sucedidos por paquetes grano-estrato decrecientes, generados durante un ciclo completo de descarga hiperpícnica (aceleración-máxima eficiencia-desaceleración), o bien por paquetes grano-estrato decrecientes que representan la etapa de aceleración-desaceleración de una descarga hiperpícnica. Ambos tipos de arreglos muestran pasajes recurrentes entre diferentes estructuras sedimentarias producidas por sedimentación continua, con intergradaciones entre zlif tractiva, maciza y erosiva. En general, las hiperpicnitas muestran asociaciones de trazas fósiles más empobrecidas que las que se han reconocido en las facies deltaicas, con dominio de estructuras de organismos suspensívoros, poco desarrollo de la estructura de escalonamiento y dominio de estrategias oportunistas.

- Los principales factores que controlaron la distribución y diversidad de las comunidades bentónicas fueron las fluctuaciones en la salinidad, la turbidez y las altas tasas de sedimentación generadas durante la descarga de los flujos hiperpícnicos. El desarrollo de asociaciones icnológicas de baja diversidad o la ausencia de las mismas en los sistemas de canales hiperpícnicos, refuerza la interpretación de que en los ambientes proximales se dieron las mayores condiciones de estrés salino, mientras que en posiciones distales del sistema con desarrollo de lóbulos hiperpícnicos, ocurre la mayor diversidad icnológica.

- El carácter fluvio-derivado de los depósitos se evidencia por la presencia de estructuras sedimentarias unidireccionales, con abundante contenido de materia orgánica y fango acumulado en las caras de avalancha de estructuras tractivas de diferente escala. En este caso, y a diferencia de las estructuras mareales donde las cortinas de fango se producen por decantación durante etapas de baja energía de la marea (slack water), la presencia de estos delgados niveles de fangos y materia orgánica conjuntamente con granos de areniscas en las caras de avalancha de las estructuras tractivas, se vincula a procesos de floculación generados bajo condiciones energéticas variables. El reconocimiento de este tipo de estructuras asociadas a niveles con presencia de materia orgánica (fragmentos de hojas y troncos) distribuidas de manera caótica en paquetes de areniscas macizas, indican una marcada vinculación con descargas fluvio-derivadas (normales y de crecida), más que con procesos de difusión mareal activos. En estos depósitos tampoco se han reconocido sistemas de galerías con rellenos que indiquen ritmos mareales; por el contrario, los sistemas de galerías muestran rellenos de areniscas o conglomerados finos sin ordenamiento interno y con abundante contenido de materia orgánica.

- El modelo depositacional desarrollado para la Formación Lajas presenta un caso sumamente interesante en el cual se reconoce una interacción entre sistemas deltaicos fluvio-dominados y descargas hiperpícnicas, a partir de un análisis sedimentológico e icnológico detallado. También brinda criterios diagnósticos que permiten diferenciar entre estructuras sedimentarias típicamente vinculadas a procesos de difusión mareal (e.g., tidal bundles), de otras similares pero producidas por descargas fluviales de diferente magnitud. Estas características pueden resultar relevantes a la hora de caracterizar el tipo de sistema deltaico y sus variaciones temporales y espaciales, permitiendo de este modo hacer predicciones sobre la distribución y espesor de sus principales reservorios.

\section{Agradecimientos}

Los autores desean expresar su agradecimiento al Dr. C. Zavala por la revisión crítica y los oportunos comentarios realizados a una versión preliminar de este trabajo, y al Dr. D. Martinioni por la lectura crítica y sus comentarios al manuscrito final. Se agradece a los 
Drs. Buatois, Spalletti y Lara, la revisión crítica y los comentarios aportados al manuscrito. Este estudio fue financiado por CONICET PIP 417 y Proyecto de Vinculación Tecnológica RSPU 3270/13 a JJP, PICT 2011-1373 y PICTO-UNRN 2010-0199 a NBC. Se agradece también a la Secretaría de Ciencia y Técnica de la Universidad Nacional del Sur por el financiamiento económico otorgado con el proyecto PGI 24/H104, y a la directora del mismo, Dra. M.E. Quattrocchio. Los autores también desean agradecer el apoyo brindado por el Instituto de Investigación en Paleobiología y Geología de la Universidad Nacional de Río Negro, el Instituto Geológico del Sur (INGEOSUR), y el Departamento de Geología de la Universidad Nacional del Sur.

\section{Referencias}

Arregui, C.; Carbone, O.; Leanza, H.A. 2011. Contexto tectosedimentario. In Congreso Geológico Argentino, No. 18 (Leanza, H.A.; Arregui, C.; Carbone, O.; Danieli, J.C.; Vallés J.M.; editores). Relatorio Geología y Recursos Naturales de la Provincia del Neuquén: 29-36. Neuquén.

Bhattacharya, J.P. 2006. Deltas. In Facies Models revisited (Posamentier, H.W.; Walker, R.G. editors). Society of Economic Paleontologists and Mineralogists, Special Publication 84: 237-292.

Branney, M.J.; Kokelaar, B.P. 1992. A reappraisal of ignimbrite emplacement: progressive aggradation and changes from particulate to non-particulate flow during emplacement of high-grade ignimbrite. Bulletin of Volcanology 54: 504-520.

Branney, M.J.; Kokelaar, B.P. 2002. Pyroclastic Density Currents and the Sedimentation of Ignimbrites. Geological Society, Memoirs 27: 137 p. London.

Buatois, L.A.; Saccavino, L.L.; Zavala, C. 2011. Ichnologic signatures of hyperpycnal flow deposits in Cretaceous river-dominated deltas, Austral Basin, southern Argentina. In Sediment transfer from shelf to deep water-Revisiting the delivery system (Slatt, R.M.; Zavala, C.; editors). American Association of Petroleum Geologists Studies in Geology 61: 153-170.

Canale, N.; Ponce, J.J. 2012. Sedimentología de sistemas de lóbulos y canales hiperpícnicos en la Formación Lajas, área de Portada Covunco, Neuquén, Argentina. Reunión Argentina de Sedimentología, No. 13, Resúmenes: p. 48. Salta.

Carmona, N.B.; Ponce, J.J. 2011. Ichnology and sedimentology of Miocene hyperpycnites of the Austral foreland basin (Tierra del Fuego, Argentina): Trace fossil distribution and paleoecological implications. In Sediment transfer from shelf to deep water-Revisiting the delivery system (Slatt, R.M.; Zavala, C.; editors). American Association of Petroleum Geologists Studies in Geology 61:171-192.

Davis, R.A. 2012. Tidal signatures and their preservation potential in stratigraphic sequences (Davis, R.A. Jr.; Dalrymple, R.W.; editors). Principles of Tidal Sedimentology: 35-55.

Domínguez, J.M.L. 1996. The São Francisco strandplain: a paradigm for wave-dominated deltas? In Geology of Siliciclastic Shelf Seas (De Baptist, M.; Jacobs, P.; editors). Geological Society, Special Publications 117: 217-231. London.

Galloway, W.E. 1975. Process framework for describing the morphologic and stratigraphic evolution of deltaic depositional systems. In Deltas, Models for Exploration (Broussard, M.L.; editor). Houston Geological Society: 87-98. Houston, Texas.

Gingras, M.K.; Bann, K.L.; MacEachern, J.A.; Waldron, W.; Pemberton, S.G. 2007. A conceptual framework for the application of trace fossils. In Applied ichnology (MacEachern, J.A.; Bann, K.L.; Gingras, M.K.; Pemberton, S.G.; editors). Society of Economic Paleontologists and Mineralogists, Short Course Notes 52: 1-25.

Groeber, P. 1946. Observaciones geológicas a lo largo del meridiano $70^{\circ}$. Hoja Chos Malal. Revista de la Sociedad Geológica Argentina: 177-208. Buenos Aires.

Gulisano, C.A. 1981. El ciclo Cuyano en el norte de Neuquén y sur de Mendoza. In Congreso Geológico Argentino, No. 8, Actas 3: 579-592. Buenos Aires.

Gulisano, C.A.; Gutiérrez-Pleimling, A.R. 1995. The Jurassic of the Neuquen Basin, a) Neuquen Province. In International Congress on Jurassic Stratigraphy and Geology, No. 4, Field guide, Serie E, Asociación Geológica Argentina: p.111. Buenos Aires.

Gulisano, C.A.; Gutiérrez-Pleimling, A.R.; Digregorio, R.E. 1984. Esquema estratigráfico de la secuencia jurásica del oeste de la provincia de Neuquén. In Congreso Geológico Argentino, No. 9, Actas 1: 236-259. San Carlos de Bariloche.

Kineke, G.C.; Woolfe, K.J.; Kuehl, S.A.; Milliman, J.D.; Dellapenna, T.M.; Purdon, R.G. 2000. Sediment export from the Sepik River, Papua New Guinea: evidence for a divergent sediment plume. Continental Shelf Research 20 (16): 2239-2266.

Leanza, H.A.; Hugo, C.A. 2001. Hoja Geológica Zapala, Hoja 3969-I, 1:250.000. Instituto de Geología y Recursos Minerales, Boletín 275: 1-128. 
Legarreta, L.; Gulisano, C.A. 1989. Análisis estratigráfico secuencial de la Cuenca Neuquina (Triásico superior-Terciario inferior, Argentina). In Cuencas Sedimentarias Argentinas (Chebli, G.; Spaletti, L.; editores). Serie Correlación Geológica, Universidad Nacional de Tucumán 6: 221-243.

Macdonald, R.G.; Alexander, A.J.; Bacon, J.C.; Cooker, M.J. 2009. Flow patterns, sedimentation and deposit architecture under a hydraulic jump on a non-eroding bed: defining hydraulic-jump unit bars. Sedimentology 56: 1346-1367.

MacEachern, J.A.; Bann, K.L.; Bhattacharya, J.P.; Howell, C.D. 2005. Ichnology of deltas: organism responses to the dynamic interplay of rivers, waves, storms and tides. In River Deltas: Concepts, Models and Examples. (Bhattacharya, J.P.; Giosan, L.; editors). Society of Economic Paleontologists and Mineralogists, Special Publication 83: 49-85.

McIlroy, D. 2004. Ichnology and facies model of a tide-dominated delta: Jurassic upper Ror and lle Formations of Kristin Field, Halten Terrace, Offshore Mid-Norway. In The Application of Ichnology to Palaeoenvironmental and Stratigraphic Analysis (McIlroy, D.; editor). Geological Society, Special Publications 228: 237-272. London.

McIlroy, D.; Flint, S.; Howell, J.A.; Timms, N. 2005. Sedimentology of the Jurassic tide-dominated Lajas Formation, Neuquén Basin, Argentina. In The Neuquén Basin, Argentina: A case study in sequence stratigraphy and basin dynamics (Veiga, G.D.; Spalletti, L.A.; Howell, J.A.; Schwarz, E.; editors). Geological Society, Special Publications 252: 83-108. London.

Mpodozis, C.; Ramos, V.A. 1989. The Andes of Chile and Argentina. In Geology of the Andes and its relation to Hydrocarbon and Mineral Resources. (Ericksen, G.E.; Cañas, M.T.; Reinemud, J.A.; editors). Circumpacific Council for Energy and Mineral Resources, Earth Science Series 11: 59-90.

Mulder, T.; Syvitski, J.P.M. 1995. Turbidity currents generated at river mouths during exceptional discharges to the world oceans. Journal of Geology 103: 285-299.

Mulder, T.; Syvitski, J.P.M. 1996. Climatic and morphologic relationships of rivers. Implications of sea levels fluctuations on river loads. Journal of Geology 104: 509-523.

Nemec, W. 1995. The dynamics of deltaic suspension plumes. In Geology of Deltas (Oti, M.N.; Postma, G.; editors). Balkema: 31-93. Rotterdam.

Olariu, C.; Bhattacharya, J.P. 2006, Terminal distributary channels and delta front architecture of fluvial- dominated delta systems. Journal of Sedimentary Research 76: 212-233.

Parsons, J.D.; Bush, J.; Syvitski, J.P.M. 2001. Hyperpycnal flow formation with small sediment concentrations. Sedimentology 48: 465-478.

Petter, A.L.; Steel, R.J. 2006. Hyperpycnal flow variability and slope organization on an Eocene shelf margin, Central Basin, Spitsbergen. American Association of Petroleum Geologists, Bulletin 90: 1451-1472.

Ponce, J.J. 2009. Análisis estratigráfico secuencial del Cenozoico de la Cordillera Fueguina, Tierra del Fuego, Argentina. Tesis Doctoral (Inédito), Universidad Nacional del Sur: 245 p.

Ponce, J.J.; Carmona, N.B. 2011, Miocene deep-marine hyperpycnal channel levee complexes, Tierra del Fuego, Argentina: Facies associations and architectural elements. In Sediment transfer from shelf to deep water-Revisiting the delivery system (Slatt, R.M.; Zavala, C.; editors), American Association of Petroleum Geologists, Studies in Geology 61: 75-93.

Ponce, J.J.; Olivero, E.; Martinioni, D.R.; López, M.I. 2007. Sustained and episodic gravity flow deposits and related bioturbation patterns in Paleogene turbidites (Tierra del Fuego, Argentina). In Sediment-Organism Interactions: A Multifaceted Ichnology. (Bromley, R.G.; Buatois, L.A.; Mángano, M.G.; Genise, J.F.; Melchor, R.N.; editors). Society of Economic Paleontologists and Mineralogists, Special Publications 88: 253-266.

Ponce, J.J.; Olivero, E.B.; Martinioni, D.R. 2008. Upper Oligocene-Miocene clinoforms of the foreland Austral Basin of Tierra del Fuego, Argentina: sequence stratigraphy and architecture of the foredeep deposits. Journal of South American Earth Sciences 26: 36-54.

Ponce, J.J.; Canale, N.; Carmona, N.B.; Bournod, C.N.; Martínez, M.A.; Olivera, D.E. 2012. Sedimentología e icnología en sucesiones hiperpícnicas de la Formación Lajas, área de Portada Covunco, Neuquén, Argentina. In Reunión Argentina de Sedimentología, No. 13, Resúmenes: 175-176. Salta.

Saccavino, L.; Buatois, L.; Zavala, C. 2008. Integrating sedimentologic and ichnologic data in characterization of hyperpycnal flow deposits of Cretaceous fluvio-dominated deltas (Magallanes Basin, southern Argentina). In American Association of Petroleum Geologists Hedberg Conference. Sediment Transfer from Shelf to Deepwater-Revisiting the Delivery Mechanisms. Ushuaia-Patagonia. 
Schieber, J.; Southard, J.; Thaisen, K. 2007. Accretion of mudstone beds from migrating floccule ripples. Science 318: 1760-1763.

Spalletti, L.A.; Franzese, J.R.; Veiga, G.D.; Schwarz, E. 2005. The Mesozoic evolution of the Neuquén Basin and its relationship with the history of Western Gondwana. In Gondwana Conference, No. 12, Abstracts: p. 343. Mendoza.

Savrda, C.E.; Bottjer, D.J. 1987. The exaerobic zone, a new oxygendeficient marine biofacies. Nature 327: 54-56.

Savrda, C.E.; Bottjer, D.J. 1989. Trace fossil model for reconstructing oxygenation histories of ancient marine bottom waters: Application to Upper Cretaceous Niobrara Formation, Colorado. Palaeogeography, Palaeoclimatology, Palaeoecology 74: 49-74.

Syvitski, J.P.M.; Morehead, M.D. 1999. Estimating riversediment discharge to the ocean: application to the Eel margin, Northern California. Marine Geology 154: 13-28.

Tunik, M.A.; Folguera, A.; Naipauer, M.; Pimentel, M.; Ramos, V. 2010. Early uplift and orogenic deformation in the Neuquén basin: Constraints on the andean uplift from $\mathrm{U}-\mathrm{Pb}$ and $\mathrm{Hf}$ isotopic data of detrital zircons. Tectonophysics 489: 257-273.

Wetzel, A. 2008. Recent bioturbation in the deep South China Sea: an uniformitarian ichnologic approach. Palaios 23: 601-615.

Wetzel, A.; Carmona, N.B.; Ponce, J.J. 2013. Tidal signature recorded by burrow fill: an example from the Miocene of Patagonia. In Ichnofabric Workshop, No. 12, Abstract: p 33. Canakkale.
Wetzel, A.; Carmona, N.B.; Ponce, J.J. 2014. Tidal signature recorded by burrow fill. Sedimentology 61: 1198-1210.

Yrigoyen, M.R. 1991. Hydrocarbon resources of Argentina. In World Petroleum Congress, No. 13. Petrotecnia, Special Issue: 38-54. Buenos Aires.

Zavala, C. 2002. El contacto entre los grupos Cuyo y Lotena (Jurásico) en la Sierra de la Vaca Muerta. Cuenca Neuquina, Argentina (Cabaleri, N.; Cingolani, C.A.; Linares, E.; López de Luchi, M.G.; Ostera, H.A.; Panarello, H.O.; editores). In Congreso Geológico Argentino, No. 15, Actas: 711-715.

Zavala, C.; González, R. 2001. Estratigrafía del Grupo Cuyo (Jurásico inferior-medio) en la Sierra de la Vaca Muerta, Cuenca Neuquina. Boletín de Informaciones Petroleras 65: 52-64.

Zavala, C.; Ponce, J.J.; Arcuri, M.; Drittanti, D.; Freije, H.; Asensio, M. 2006. Ancient Lacustrine hyperpycnites: a depositional model from a case study in the Rayoso Formation (Cretaceous) of west-central Argentina. Journal of Sedimentary Research 76: 40-58.

Zavala, C.; Arcuri, M.; Gamero, H.; Contreras, C.; Di Meglio, M. 2011. A genetic facies tract for the analysis of sustained hyperpycnal flow deposits. In Sediment transfer from shelf to deep water-Revisiting the delivery system (Slatt, R. M.; Zavala, C.; editors). American Association of Petroleum Geologists, Studies in Geology 61: 31-51.

Zavala, C.; Arcuri, M.; Blanco-Valiente, L. 2012. The importance of plant remains as a diagnostic criteria for the recognition of ancient hyperpycnites. Revue de Paléobiologie, Genéve. Volume spécial 11: 457-469.

Manuscript received: April 14, 2014; revised/accepted: December 05, 2014; available online: December 09, 2014. 